\title{
Importance of confinement in instanton induced potential for bottomonium spectroscopy
}

\author{
Bhoomika Pandya ${ }^{1, \mathrm{a}}$ (D), Manan Shah ${ }^{2, \mathrm{~b}}$, P. C. Vinodkumar ${ }^{1, \mathrm{c}}$ \\ ${ }^{1}$ Department of Physics, Sardar Patel University, Vallabh Vidyanagar, Gujarat 388120, India \\ 2 P. D. Patel Institute of Applied Sciences, CHARUSAT, Changa, India
}

Received: 21 October 2020 / Accepted: 22 January 2021 / Published online: 3 February 2021

(c) The Author(s) 2021

\begin{abstract}
Mass spectra of bottomonium states are computed using the Instanton Induced potential obtained from Instanton Liquid Model for QCD vacuum and incorporating a stronger confinement term. Spin dependent interactions through confined one gluon exchange potential are incorporated to remove the mass degeneracy. The mass spectra of the $b \bar{b}$ states up to $4 S$ states are found to be in good agreement with the values reported by PDG(2020). Mixing of nearby isoparity states are also studied. We found the state $\Upsilon(10,860)$ as an admixture of $5^{3} S_{1}$ and $6^{3} D_{1}$ Upsilon states with mixing angle $\theta=39.98^{\circ}$ and the mixed state di-leptonic decay width is found to be $0.25 \mathrm{keV}$ as against the width of $0.31 \pm 0.07 \mathrm{keV}$ reported by PDG. Further the state $\Upsilon(11,020)$ is also found to be the admixture of $6^{3} S_{1}$ and $5^{3} D_{1}$ Upsilon states with the mixing angle $\theta=51.69^{\circ}$ and the di-leptonic decay width of the mixed state is obtained as $0.14 \mathrm{keV}$ which is very close to the width of $0.13 \pm 0.03 \mathrm{keV}$ reported by PDG. Present results indicates that addition of confinement to the instanton potential is crucial for the determination of the mass spectroscopy of heavy hadrons.
\end{abstract}

\section{Introduction}

Quarkonia are regarded as the simple and appropriate hadronic systems to explore the QCD aspects at the low energy regimes through its spectroscopy [1-4]. Since the discovery of $\Upsilon(1 S), \Upsilon(2 S)$ and $\Upsilon(3 S)$ at the Fermilab by E288 collaboration $[5,6]$; many of its orbital excited states such as $\chi_{b 0,1,2}(1 P)$ and $\chi_{b 0,1,2}(2 P)$ etc. are also discovered subsequently [7-10]. Further, continuous progress has been achieved in the bottomonium spectroscopy by the discovery of $\Upsilon(4 S), \Upsilon(10,860)$ and $\Upsilon(11,020)[11,12]$. BABAR

\footnotetext{
a e-mail: bhumispandya@gmail.com (corresponding author)

b e-mail: mnshah09@gmail.com

c e-mail: p.c.vinodkumar@gmail.com
}

collaboration discovered the spin singlet low lying $\eta_{b}(1 S)$ [13] and by the efforts of CLEO, Belle and BABAR its average mass is found to be $9398.7 \pm 2.0 \mathrm{MeV}$ [14-16]. CLEO has given the successful observation of the $\eta_{b}(2 S)$ with $9974.6 \pm 2.3 \pm 2.1 \mathrm{MeV}$ [17] but BELLE recorded the state having a mass of $9999.0 \pm 3.5 \mathrm{MeV}$ [18] after performing the experiment by taking much larger data sets than [17]. $\Upsilon\left(1^{3} D_{2}\right)$ also well established $[19,20]$ and the low lying $h_{b}(1 P)$ and $h_{b}(2 P)$ are also reported by BABAR [21]. Recently, LHCb collaboration found the mass of $\chi_{b 1}(3 P)$ as $10,515_{-3.9}^{+2.2} \mathrm{MeV}$ [22]. Two other charged states in the bottom family, $Z_{b}(10,610)$ and $Z_{b}(10,650)$ are also being reported recently [23]. Their electric charge suggest that they do not belong to conventional bottomonium $[24,25]$. So we do not include them in the present work.

Looking to the advances in the experimental side, it is necessary to review earlier theoretical attempts to understand the quarkonia systems. Although there are many different approaches like Lattice QCD methods [26-29], NRQCD methods [30-32], Light front quark model [33-36], various coupled channel quark models [37-39], Effective Lagrangian approach [40] etc., employed to study them, still there exist no consensus and discrepancy persist in the predictions. For instance, authors [41] have used nonrelativistic constituent quark model wherein the lowest lying bottomonium states $\Upsilon(1 S)$ and $\eta_{b}(1 S)$ are about $50 \mathrm{MeV}$ higher than the PDG [43] data but the same model give fine agreement for the higher excited state $\Upsilon(6 S)$ while the relativized quark model [42] provides very good description of the low-lying states but higher excited $\Upsilon(6 S)$ is overestimated by $100 \mathrm{MeV}$.

It is believed that most reliable description of the bound system can be obtained from Bethe-Salpeter formalism. It is a relativistic quantum field theory but ambiguity is that the interaction kernel is not solvable from QCD and it becomes very tough to extract hadronic information [44]. In EFTs, threshold region is still disappointing [46]. Lattice regularized QCD beyond threshold and full understanding of bot- 
tomonium seems difficult [47-54] even though very recently they have predicted few radial excitations.

Currently, computational complexity of other theoretical approaches makes the potential model approach as the most reliable which is able to meet the expectations. Potential models provide quantitative and qualitative analysis of the quarkonia states. Potential models account for both the shorter and larger distance behaviour of the $q-\bar{q}$ interaction. The most promising non-perturbative contribution is the "Quark Confinement" which can be incorporated by the "Wilson loop". Such non-perturbative effects will increase at the larger distances $[2,55]$. And such attempts are key to the lattice QCD calculations. Apart from the Wilson loop, the contribution from Instantons [56] is also considered for the non-perturbative effects at the larger distance. Instantons, which are the large fluctuations of the gluon field and corresponds to the tunneling from one minimum of the energy to the neighbouring one. Similar fluctuations but having tunneling in the opposite side is called "anti-instantons".

In the present work we aim to find the effect of the instantons on the heaviest quarkonium system. As the form of the instanton potential contains the term which gives the non-perturbative effect at the larger distance as well as coloumbian type behaviour of the shorter distance together make it suitable for the study of the spectroscopy of bottomonium. Additionally, we have also incorporated the spin dependent part of one gluon exchange potential. The detailed description of the form of instanton potential for heavy quark can be found in [57] where authors have added the spin dependent attributes into it. This potential has a long history [5961] and its central part was derived long ago [62] based on the instanton liquid model $[63,66,67]$ for the QCD vacuum. With the use of instanton potential acting between quark and antiquark pair, we solved the non-relativistic Schrödinger equation by variational method. To test the predictivity of the potential we have further calculated various decay properties like vector and pseudoscalar decay constants, di-leptonic, digamma, di-gluon as well as tri-gluon decays together with radiative transition decay widths. The computed results are tabulated and compared with other available theoretical and experimental data and finally we draw important conclusions.

\section{Theoretical framework to compute mass spectra}

Evaluation of the bound state mass spectra demands the solution of the Schrödinger equation with the potential from the instanton vacuum. Unfortunately, the Schrödinger equation is not exactly solvable for most of the systems. So, generally one adopts approximate methods like perturbation, variational or WKB methods. In the present work, the variational method is employed to calculate the ground and excited state mass spectra. For the treatment of bottomonium on the non- relativistic footing the Hamiltonian can be written as

$H=\frac{P^{2}}{2 M}+V(r)$

Here, $P$ is the relative momenta, $M$ represents reduced mass, $V(r)$ is the potential acting between quark and antiquark. The QCD instanton effects are important to apprehend the phenomena in the non-perturbative region in QCD. It is believed that instantons are present in the QCD vacuum and they are working effectively in reproducing many remarkable features of the strong interactions [64]. The most significant success of instantons is their capacity to provide a microscopic mechanism of the spontaneous chiral symmetry breaking $[64,65]$. But there exist lack of successful numerical predictions of the mass spectroscopy and decay properties based on the instanton inspired models. A recent attempt by Yakhshiev et al. [57] have obtained the mass spectra of low lying quarkonium states. For the case of ground state of bottomonium they found differences of $1006 \mathrm{MeV}$ and $945 \mathrm{MeV}$ for $\Upsilon(1 S)$ and $\eta_{b}(1 S)$ respectively as compared to PDG. For the $(1 P)$ state difference was more than $1200 \mathrm{MeV}$. Also, the hyperfine contribution was very much less in their study as compared to the experimental mass splittings between spin triplet and spin singlet states. According to Schäfer and Shuryak in a review paper on "Instanons in QCD", it is clearly stated that for heavy flavour hadrons and for higher states, the confinement effect play an important role [58] following this suggestions from [58] and looking at the results of the attempt made by [57] we found that stronger confinement effects are needed to improve the results. Thus, we have chosen instanton potential incorporating additional confinement part in the potential to predict the mass spectra and other decay properties in the bottom sector. So, for present study the form of the potential we have used is instanton induced potential according to the Instanton Liquid Model (ILM) [57]. The ILM has two important parameters portraying the diluteness of the instanton liquid $[63,68]$ : one is the average size of instanton $(\bar{\rho})$ and other is the average distance between instantons $(\bar{R})$. Numerical values of these parameters are different for different approaches. For example, Shuryak [68] proposed the values of these parameters as $\bar{\rho} \simeq 0.33 \mathrm{fm}$ and $\bar{R} \simeq 1 \mathrm{fm}$. In ref [69-71], $\bar{\rho} \simeq 0.35 \mathrm{fm}$ and $\bar{R} \simeq 0.856 \mathrm{fm}$ were used in their $1 / N_{c}$ meson loop contibution for the light quark sector. And in this framework the range of the potential is identified only by the size of the instanton $(\bar{\rho})$.

For $r \ll \bar{\rho}$ i.e., when the distance between quarkantiquark is smaller than the average size of instanton, the central potential is given by [57]

$$
V(r) \simeq \frac{4 \pi \bar{\rho}^{3}}{\bar{R}^{4} N_{c}}\left(1.345 \frac{r^{2}}{\bar{\rho}^{2}}-0.501 \frac{r^{4}}{\bar{\rho}^{4}}\right)
$$

here, $N_{c}=3$ represents the colour degrees of freedom. 
For $r \gg \bar{\rho}$ i.e., the distance between quark-antiquark is higher than the size of instanton, the central part of the potential is given by [57]

$V(r) \simeq 2 \Delta M_{Q}-\frac{g_{n p}}{r}$

here, $\Delta M_{Q}$ is the correction to heavy-quark mass from the instanton vacuum and is given by [57].

$\Delta M_{Q}=-\frac{4 \pi^{4} \bar{\rho}^{3}}{3 \bar{R}^{4} N_{c}}\left(J_{0}(\pi)+\frac{1}{\pi} J_{1}(\pi)\right)$

where, $J_{0}$ and $J_{1}$ are the Bessel functions. The coupling constant in Eq. (3) is defined as $g_{n p}=2 \pi^{3} \bar{\rho}^{4} / N_{c} \bar{R}^{4}$ and can be considered as nonperturbative correction to the strong coupling constant $\alpha_{S}(r)$. When $r$ reaches to infinity, the potential is saturated at $2 \Delta M_{Q}$ and it signifies that instanton vacuum cannot explain quark confinement [62]. Thus for the present study, we have added a state dependent confinement potential $V_{0}$ which has the form

$$
\begin{aligned}
V_{0}(z) & =b \ln (z)+a \\
& =b \ln (2 N+l-1)+a
\end{aligned}
$$

Where, $z=(2 N+3)-(4-l)$ and $N=2 n+l$. Here, $n$ is the radial quantum number and $l$ is the orbital quantum number. $z$ represents the eigenvalues corresponds to modified harmonic oscillator like potential. The modification can also be attributed to the centre of mass corrections which is state dependent. $V_{0}$ is then a logarithmically dependent eigenvalue corresponds to a modified three dimensional harmonic oscillator potential. The parameters $a$ and $b$ are our fitted model parameters which are deduced by fitting the masses of low lying bottomonium states based on instanton potential framework. The numerical values of the constants are $a=211.1 \mathrm{MeV}$ and $b=290.48 \mathrm{MeV}$. For instanton liquid model framework, potential is defined only for $r \ll \bar{\rho}$ and $r \gg \bar{\rho}$ and to remove the discontinuity at $r=\bar{\rho}$ we have further added $V_{1}=61.81 \mathrm{MeV}$ to the long range part of the interaction represented by Eq. (3). It is justifiable from the QCD point of view that hadronic bound states has contributions from the long range part of the interactions. This inclusion changes the potential range from $r \ll \bar{\rho}$ to $r \leq \bar{\rho}$ and $r \gg \bar{\rho}$ to $r \geq \bar{\rho}$. The instanton potential parameters as $\bar{\rho} \simeq 0.36 \mathrm{fm}$ and $\bar{R} \simeq 0.89 \mathrm{fm}$ from the lattice simulation study of Instanton vacuum [72,73] are being used.

Figure 1 shows the potential as a function of radial distance for few low lying states incorporating the addition of $61.81 \mathrm{MeV}$ to Eq. (3). Exclusion of this addition of 61.81 MeV to Eq. (3) leads to kink at $r=0.36 \mathrm{fm}$ in the behaviour of the potential. The potential corresponds to the instanton liquid model as a continuous function of the radial separation with and without the state dependent confinement part are plotted in Fig. 1. It is to be noticed that the addition of confinement provides a shift to the potential while retaining the shape.

The harmonic oscillator trial wave-function used here is

$$
R_{n l}(r)=N_{n l}(\mu r)^{l} \exp \left(-\mu^{2} r^{2} / 2\right) L_{n-1}^{l+1 / 2}\left(\mu^{2} r^{2}\right)
$$

with $N_{n l}$ being the normalization constant expressed as

$$
N_{n l}=\sqrt{\frac{2 \mu^{3}(n-1) !}{\Gamma(n+l+1 / 2)}}
$$

Also, $\mu$ is the variational parameter and $L_{n-1}^{l+1 / 2}\left(\mu^{2} r^{2}\right)$ is the associated Laguerre polynomial.

We employ the Ritz variational method by minimizing the expectation value of the Hamiltonian to obtain the variational parameter $\mu$ corresponds to the ground state. In the case of excited states, we have employed the Virial theorem $\left(\langle T\rangle=\frac{1}{2}\left\langle r \frac{\partial V(r)}{\partial r}\right\rangle\right)$ to estimate the variational parameter. The deduced variational parameter correspond to few lowlying states are given in Table 1. Based on the above theoretical formalism, we have computed the spectroscopic masses of the $S, P$ and $D$ wave bottomonium. The spin averaged masses of the radial $(n S)$ and orbital excited states $(n P, n D)$ are computed as

$M_{S A}=m_{b}+m_{\bar{b}}+\langle H\rangle$

Further spin dependent confined one gluon exchange interactions are used to remove the mass degeneracy of the bottomonium states. The $\mathrm{S}$ wave degeneracy is removed by introducing the hyperfine interaction given by

$V_{Q \bar{Q}}^{S S}(r)=\frac{8}{9} \frac{\alpha_{s}}{m_{Q} m_{\bar{Q}}} \mathbf{S}_{\mathbf{Q}} \cdot \mathbf{S}_{\bar{Q}} 4 \pi \delta^{3}(r)$

For the masses of $P$ and $D$ waves, we have incorporated the contribution of the spin-orbit and tensor part of the confined one gluon exchange potential (COGEP) of the form given by $[77,78,93,110-112]$

$$
\begin{aligned}
& V_{Q \bar{Q}}^{L S}(r)=\frac{\alpha_{S}}{4} \frac{N_{Q}^{2} N_{\bar{Q}}^{2}}{m_{Q^{m}} m_{\bar{Q}}} \frac{\lambda_{Q} \cdot \lambda_{\bar{Q}}}{2 r} \\
& \otimes\left[\left[\mathbf{r} \times\left(\hat{p_{Q}}-\hat{p}_{\bar{Q}}\right) \cdot\left(\sigma_{Q}+\sigma_{\bar{Q}}\right)\right]\left(D_{0}^{\prime}(r)+2 D_{1}^{\prime}(r)\right)\right. \\
& \left.+\left[\mathbf{r} \times\left(\hat{p_{Q}}+\hat{p}_{\bar{Q}}\right) \cdot\left(\sigma_{i}-\sigma_{j}\right)\right]\left(D_{0}^{\prime}(r)-D_{1}^{\prime}(r)\right)\right]
\end{aligned}
$$

and

$$
\begin{aligned}
V_{Q \bar{Q}}^{T}(r)= & -\frac{\alpha_{s}}{4} \frac{N_{Q}^{2} N_{\bar{Q}}^{2}}{m_{Q} m_{\bar{Q}}} \\
& \otimes \lambda_{Q} \cdot \lambda_{\bar{Q}}\left(\left(\frac{D_{1}^{\prime \prime}(r)}{3}-\frac{D_{1}^{\prime}(r)}{3 r}\right) S_{Q \bar{Q}}\right)
\end{aligned}
$$

where $S_{Q \bar{Q}}=\left[3\left(\sigma_{Q} \cdot \hat{r}\right)\left(\sigma_{\bar{Q}} \cdot \hat{r}\right)-\sigma_{Q} \cdot \sigma_{\bar{Q}}\right]$ with $\hat{r}=\hat{r}_{Q}-\hat{r}_{\bar{Q}}$ as the unit vector in the direction of r. $N_{Q}$ and $N_{\bar{Q}}$ are the normalization constants of the wave functions for quark and 

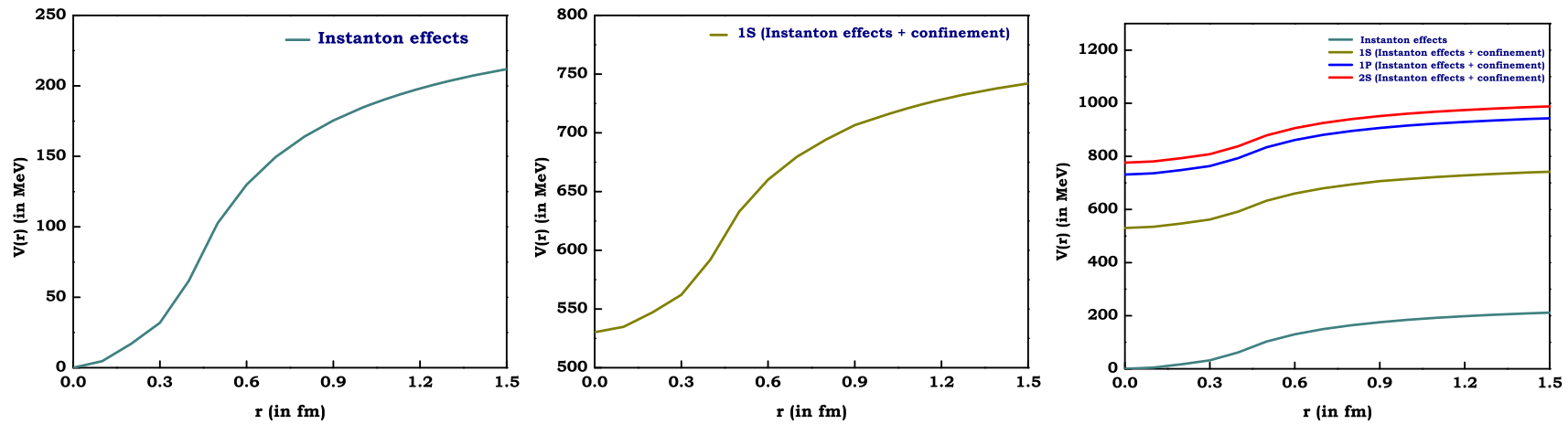

Fig. 1 The heavy quark potential from instanton vacuum for the $r \leq \bar{\rho}$ and $r \geq \bar{\rho}$ without confinement (matte green) and with confinement for $1 S$ (dark yellow), $2 S$ (red) and $1 P$ (blue)

Table 1 The variational parameter $(\mu)$, spin-average mass and expectation values of spin-dependent contribution for few low-lying bottomonium bound states employing only the instanton effects without addition of confinement (a) and instanton effects with confinement (b)

\begin{tabular}{|c|c|c|c|c|c|}
\hline State & $\begin{array}{l}\text { Variational parameter } \mu \\
\text { (in } \mathrm{MeV} \text { ) }\end{array}$ & $\begin{array}{l}M_{S A} \\
(\text { in } \mathrm{MeV} \text { ) }\end{array}$ & $\begin{array}{l}\left\langle V_{s s}\right\rangle \\
\text { (in } \mathrm{MeV} \text { ) }\end{array}$ & $\begin{array}{l}\left\langle V_{l s}\right\rangle \\
(\text { in } \mathrm{MeV})\end{array}$ & $\begin{array}{l}\left\langle V_{t}\right\rangle \\
(\text { in } \mathrm{MeV})\end{array}$ \\
\hline \multicolumn{6}{|c|}{ (a) Instanton potential without confinement term (IMWOC) } \\
\hline $1 \mathrm{~S}$ & 479.9 & 8526.19 & 2.36 & $\ldots$ & $\ldots$ \\
\hline $2 \mathrm{~S}$ & 410.6 & 8641.34 & 2.24 & $\ldots$ & $\ldots$ \\
\hline $1 \mathrm{P}$ & 460.13 & 8613.87 & $\ldots$ & 0.85 & 0.24 \\
\hline $2 \mathrm{P}$ & 384.78 & 8681.89 & $\ldots$ & 0.60 & 0.17 \\
\hline $1 \mathrm{D}$ & 432.52 & 8675.38 & $\ldots$ & 0.30 & 0.08 \\
\hline $2 \mathrm{D}$ & 368.42 & 8719.95 & $\ldots$ & 0.21 & 0.05 \\
\hline \multicolumn{6}{|c|}{ (b) Instanton potential with confinement term (IMWC) } \\
\hline $1 \mathrm{~S}$ & 1233 & 9448.61 & 48.53 & $\ldots$ & $\ldots$ \\
\hline $2 \mathrm{~S}$ & 995.2 & 10018.54 & 30.74 & $\ldots$ & $\ldots$ \\
\hline $1 \mathrm{P}$ & 1123 & 9874.56 & $\ldots$ & 7.78 & 2.35 \\
\hline $2 \mathrm{P}$ & 941.9 & $10,270.00$ & $\ldots$ & 5.43 & 1.65 \\
\hline $1 \mathrm{D}$ & 1035 & $10,153.80$ & $\ldots$ & 2.46 & 0.72 \\
\hline $2 \mathrm{D}$ & 892.6 & $10,456.6$ & $\ldots$ & 1.77 & 0.50 \\
\hline
\end{tabular}

antiquark. $\lambda_{Q} \lambda_{\bar{Q}}$ is the color factor and can be calculated separately as it is independent of the flavour contents of the quarks. For mesons $\left\langle\lambda_{i} \cdot \lambda_{j}\right\rangle_{q \bar{q}}=-\frac{4}{3}$ [115].

Expression for the confined gluon propagators are taken from $[77,93]$ as,

$$
\begin{aligned}
& D_{0}(r)=\left(\frac{\alpha_{1}}{r}+\alpha_{2}\right) \exp \left(-r^{2} c_{0}^{2} / 2\right) \\
& D_{1}(r)=\frac{\gamma}{r} \exp \left(-r^{2} c_{1}^{2} / 2\right)
\end{aligned}
$$

where, $\alpha_{1}=1.035, \alpha_{2}=0.3977 \mathrm{GeV}, c_{0}=0.3418 \mathrm{GeV}, \gamma=$ 0.8639 and $c_{2}=0.4123 \mathrm{GeV}$ are used here as the same as used in previous study of quarkonium states [93].

The strong running coupling constant $\alpha_{s}$ is calculated using the form,

$\alpha_{s}\left(M^{2}\right)=\frac{4 \pi}{\left(11-\frac{2}{3} n_{f}\right)\left(\ln \frac{M^{2}}{\Lambda^{2}}\right)}$ where, $\Lambda_{Q C D}$ is taken as $0.156 \mathrm{GeV}$ which will give the PDG [43] listed value of $\alpha_{s}$ at the $Z$ meson mass (91 GeV) range of 0.0118. Employing $\Lambda_{Q C D}=0.156 \mathrm{GeV}$ and putting spin average mass $\left(M_{S A}\right)$ of respective state in Eq. (14) gives $\alpha_{s}$ for corresponding state. Here, $n_{f}$ represents the number of flavours and for bottom sector it is 4 .

To assess the importance of confinement in the instanton models, we have computed the spin average masses of the few low-lying states of bottomonium without considering the confinement part (Eqs. (2) and (3)) and with incorporating confinement part (Eqs. (2), (3) and (5)). The results are presented here in Table 1. It is seen that the predicted low-lying spin-average masses without confinement are off by about $900 \mathrm{MeV}$ or more as found in the study reported by Ref. [57]. While the Instanton potential with confinement (IMWC) generates the spin average mass very close to the experimentally deduced values. It is observed that the average hyperfine and fine structure contribution in the case of instan- 
Table 2 S-wave mass spectrum of bottomonium bound states (in MeV). Results are compared with Experimental data [43] Relativistic Dirac model [93] Relativistic functional approach [107] Constituent quark model [41] Relativistic quark model [42] Non- relativistic potential model [101] Relativistic quark model [108] Relativistic potential model [100]

\begin{tabular}{|c|c|c|c|c|c|c|c|c|c|c|c|c|c|}
\hline $\mathrm{nS}$ & $\mathrm{M}_{S A}$ & State & $J^{P C}$ & Spin-Spin contribution & Present & PDG [43] & [93] & [107] & [41] & {$[42]$} & [101] & [108] & [100] \\
\hline \multirow[t]{2}{*}{$1 \mathrm{~S}$} & 9448.61 & $1^{3} S_{1}$ & $1^{--}$ & 12.13 & 9460.75 & $9460.30 \pm 0.26$ & 9460.99 & 9490 & 9502 & 9465 & 9460 & 9460 & 9460 \\
\hline & & $1^{1} S_{0}$ & $0^{-+}$ & -36.39 & 9412.22 & $9398.7 \pm 2.0$ & 9390.7 & 9414 & 9455 & 9402 & 9392 & 9398 & 9393 \\
\hline \multirow[t]{2}{*}{$2 \mathrm{~S}$} & $10,018.54$ & $2^{3} S_{1}$ & $1^{--}$ & 7.68 & $10,026.22$ & $10,023.26 \pm 0.31$ & $10,024.1$ & 10,089 & 10,015 & 10,003 & 10,024 & 10,023 & 10,023 \\
\hline & & $2^{1} S_{0}$ & $0^{-+}$ & -23.05 & 9995.48 & . & 9999.3 & 9987 & 9990 & 9976 & 9991 & 9990 & 9987 \\
\hline \multirow[t]{2}{*}{$3 \mathrm{~S}$} & $10,358.24$ & $3^{3} S_{1}$ & $1^{--}$ & 6.41 & $10,364.65$ & 10,3 & $10,356.2$ & 10,327 & 10,349 & 10,354 & 10,346 & 10,355 & 10,364 \\
\hline & & $3^{1} S_{0}$ & $0^{-+}$ & -19.24 & $10,339.00$ & $\cdots$ & $10,325.3$ & $\ldots$ & 10,330 & 10,336 & 10,323 & 10,329 & 10,345 \\
\hline \multirow[t]{2}{*}{$4 \mathrm{~S}$} & $10,588.98$ & $4^{3} S_{1}$ & $1^{--}$ & 5.49 & $10,594.47$ & $10,579.4 \pm 1.2$ & $10,576.2$ & $\ldots$ & 10,607 & 10,635 & 10,575 & 10,586 & 10,643 \\
\hline & & $4^{1} S_{0}$ & $0^{-+}$ & -16.48 & $10,572.49$ & $\cdots$ & $10,554.4$ & $\ldots$ & $\ldots$ & 10,523 & 10,558 & 10,573 & 10,364 \\
\hline \multirow[t]{2}{*}{$5 \mathrm{~S}$} & $10,761.30$ & $5^{3} S_{1}$ & $1^{--}$ & 4.84 & $10,766.14$ & $10,885.2_{-1.6}^{+2.6}$ & $10,758.5$ & $\ldots$ & 10,818 & 10,878 & 10,755 & 10,869 & $\cdots$ \\
\hline & & $5^{1} S_{0}$ & $0^{-+}$ & -14.53 & $10,746.76$ & $\cdots$ & $10,738.4$ & .. & $\cdots$ & 10,869 & 10,741 & 10,851 & $\ldots$ \\
\hline \multirow[t]{2}{*}{$6 \mathrm{~S}$} & $11,077.39$ & $6^{3} S_{1}$ & $1^{--}$ & 4.31 & $11,081.70$ & $11,000 \pm 4$ & $\ldots$ & $\ldots$ & 10,995 & 11,243 & 10,904 & 11,088 & $\ldots$ \\
\hline & & $6^{1} S_{0}$ & $0^{-+}$ & -12.92 & $11,064.47$ & $\cdots$ & $\ldots$ & $\ldots$ & $\ldots$ & 11,226 & 10,892 & 11,061 & $\ldots$ \\
\hline
\end{tabular}

ton model without confinement (IMWOC) is very small compared to the case with confinement. For example, the ground state $\left(1^{3} S_{1}\right)$ mass of the bottomonium system in the case of IMWOC is obtained as $8326 \mathrm{MeV}$ as against the experimental mass of $9460 \mathrm{MeV}$. Further the energy difference between $\Upsilon(1 S)$ and $\Upsilon(2 S)$ in the case of IMWOC is just $115 \mathrm{MeV}$ as against the experimental mass difference of $565 \mathrm{MeV}$. In is very clear then that, the instanton model without confinement term does not provide the masses in accordance with the experimental values. Hence, in the present study of bottomonium states we incorporate confinement along with the Instanton potential.

Results obtained here are tabulated in the Table 2 for $S$ wave masses and in Table 3 for $P$ and $D$ wave masses. We have compared our data set with the theoretical model predictions such as relativistic Dirac Model [93], QCD Relativistic functional approach [107] where authors have used two different methods rainbow-ladder truncation of DysonSchwinger and Bethe-Salpeter in search of the effects of the varying shapes of the effective running coupling on ground as well as excited states in the channels having quantum numbers $J$ less than or equal to 3 , Constituent quark model [41], Relativistic quark model [42,108], Relativistic potential model [100] and with the recent experimental data listed by PDG [43]. Also, Fig. 2 shows the energy level diagram of the bottomonium states comparing results with the PDG [43] reported values.

\section{Decay properties of heavy quarkonia $Q \bar{Q}$}

For any potential model, apart from the mass spectra other observables like radiative decays and annihilation decays are important testing ground to know the inter-quark interactions. Keeping this view, we have computed the decay properties of $b \bar{b}$ states with no additional parameters. Further, We have incorporated these decay properties with and without QCD correction.

\subsection{Pseudoscalar and vector decay constants}

Estimation of the decay constants of mesons constituting heavy quarks is essential part of the study as it offers the information of the CKM (Cabibbo-Kobayashi-Maskawa) matrix elements. The conventional formula within the nonrelativistic limit for the pseudoscalar and vector states of the $S$ wave heavy quarkonia is the Van Royen-Weiskopf formula [74] given by

$f_{P / V}^{2}(n S)=\frac{3\left|R_{n S}(0)\right|^{2}}{\pi M_{n S}} \bar{c}^{2}\left(\alpha_{S}\right)$

Where, the first order QCD correction factor $\bar{c}^{2}\left(\alpha_{S}\right)$ is expressed as $[75,76]$

$\bar{c}\left(\alpha_{s}\right)=1-\frac{\alpha_{s}}{\pi}\left(\delta^{P, V}-\frac{m_{1}-m_{2}}{m_{1}+m_{2}} \ln \frac{m_{1}}{m_{2}}\right)$

In the case of quarkonia, the second term inside the bracket in Eq. (16) vanishes. The term $\delta^{V}=8 / 3$ in the case of vector state and $\delta^{P}=2$ for the pseudoscalar state. The calculated vector and pseudoscalar decay constants with and without the first order QCD corrections are presented in Tables 4 and 5 and are compared with other available model predictions. 


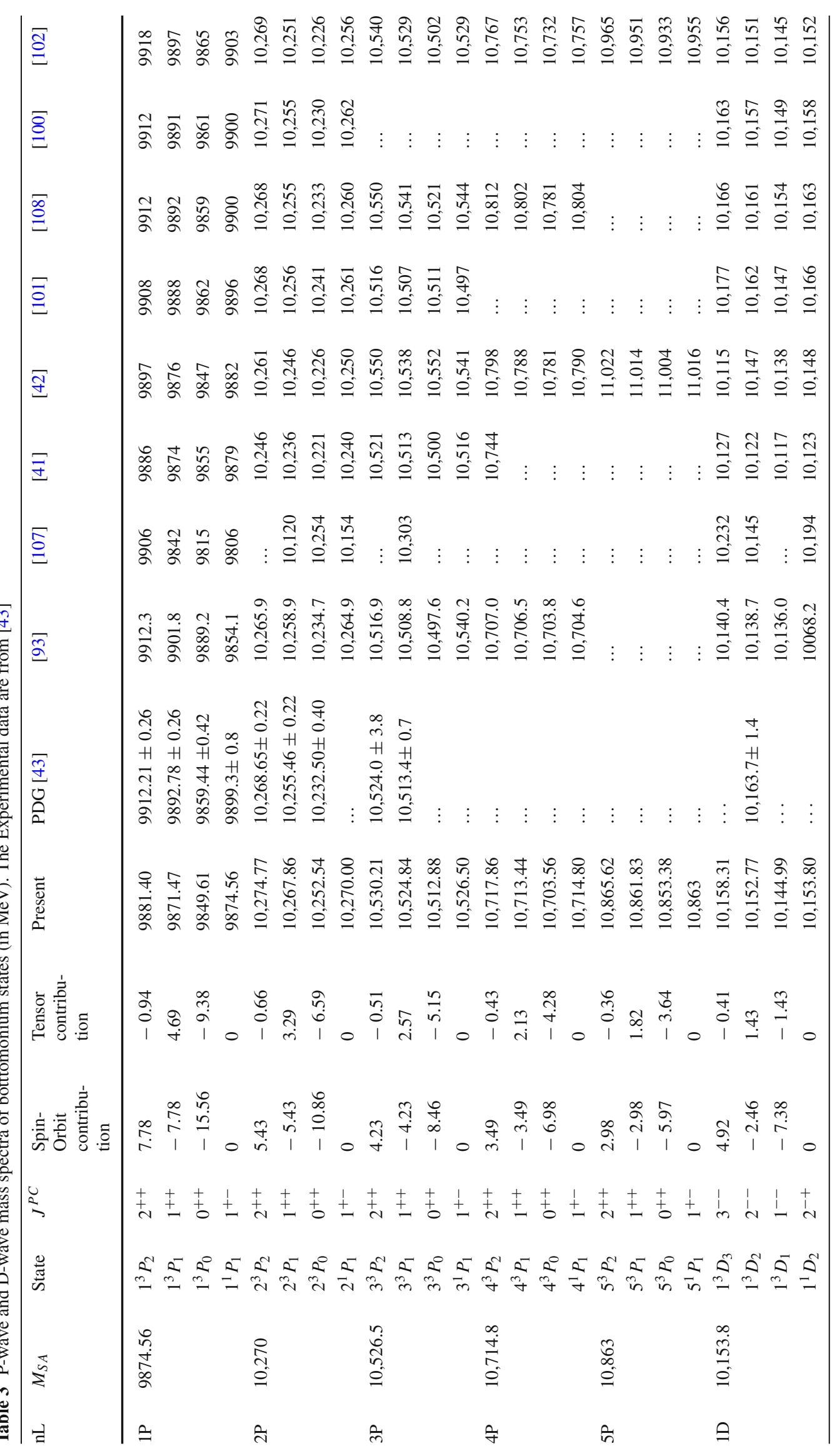




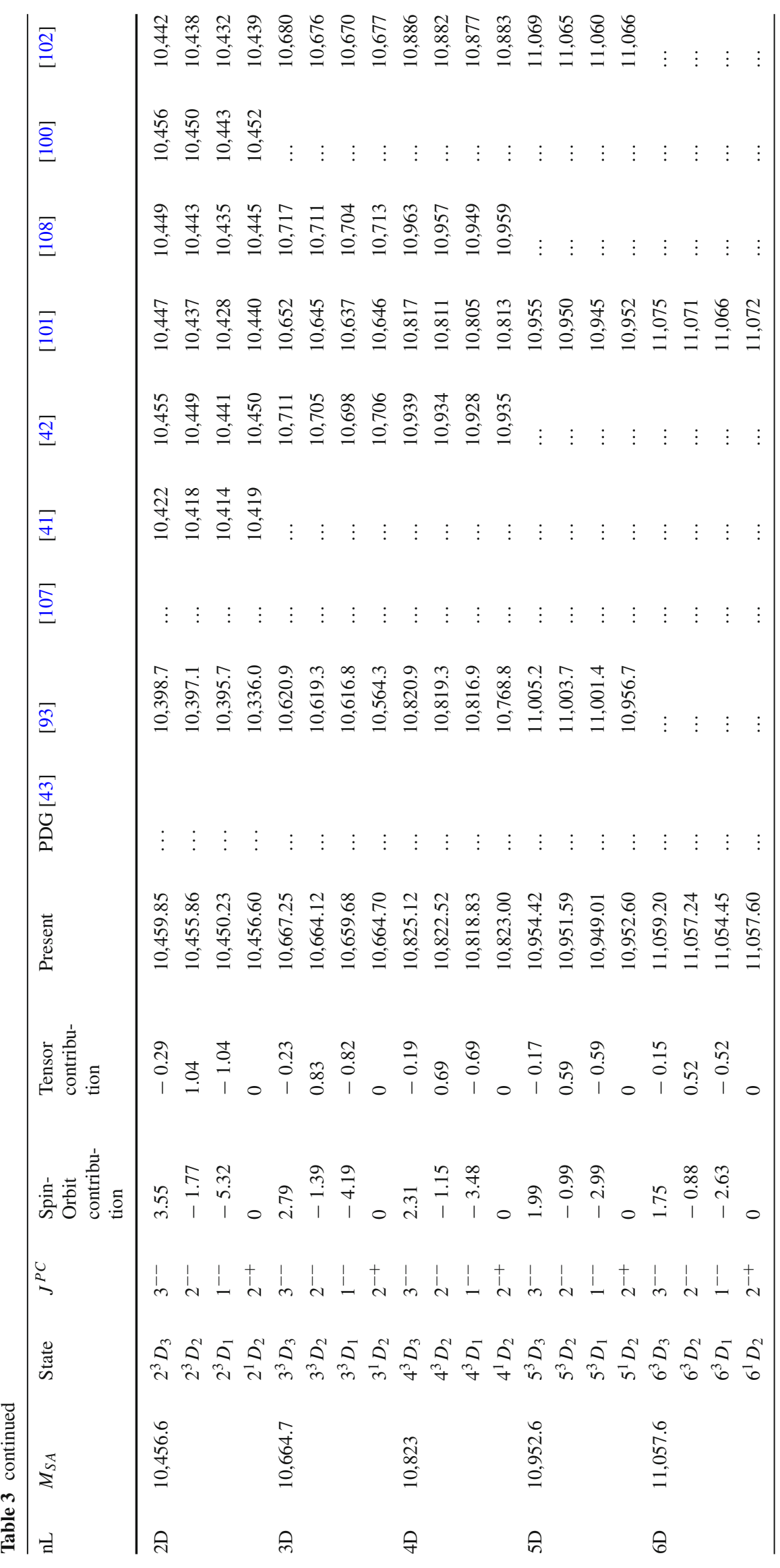




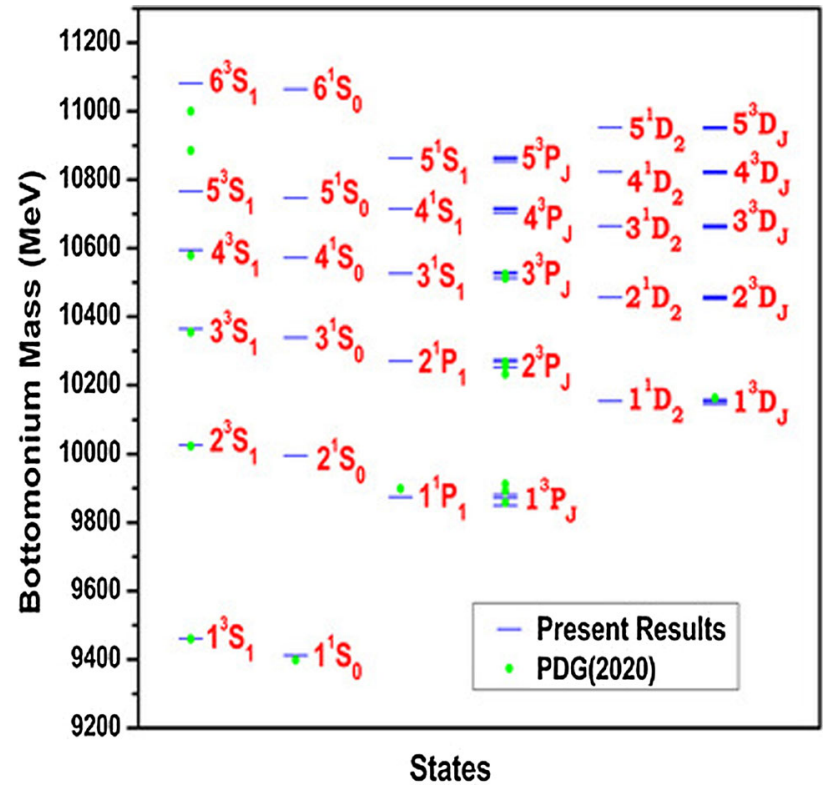

Fig. 2 Bottomonium $(b \bar{b})$ mass spectrum. Comparison of present results (blue horizontal lines) with experimental results by Particle Data Group [43] (green full circles)

\subsection{The leptonic decays of bottomonium states}

Descriptive study of leptonic decays of mesons not only provide underlying dynamics of quark anti-quark annihilation [80] but also used as a probe to study compactness of the pair of quark-antiquark bound states [79]. In addition to that leptonic decay rates can also help us to distinguish conventional mesons and exotic states [81] .

The vector mesons $\left(1^{--}\right)$annihilates leptonically via single virtual photon and leptonic partial decay width computed using Van Royen- Weisskopf formula [74] along with the QCD correction of one loop level given as [82,83]

$\Gamma\left(n^{3} S_{1} \rightarrow l^{+} l^{-}\right)=\frac{4 \alpha_{e}^{2} e_{Q}^{2}}{M^{2}\left(n^{3} S_{1}\right)}\left|R_{n l}(0)\right|^{2}\left[1-\frac{16 \alpha_{s}}{3 \pi}\right]$

Here, $\left|R_{n l}(0)\right|^{2}$ is the square of the radial wave function at the origin. $\alpha_{e}=1 / 137$ and $\alpha_{s}$ are the electromagnetic coupling constant and strong running coupling constant respectively. $e_{Q}$ is the charge of the heavy quark in units of electron charge. For the $D$ wave states $n^{3} D_{1}$ leptonic decay width can be obtained as [84],

$\Gamma\left(n^{3} D_{1} \rightarrow l^{+} l^{-}\right)=\frac{25 \alpha_{e}^{2} e_{Q}^{2}}{2 m_{b}^{4} M^{2}\left(n^{3} D_{1}\right)}\left|R_{n l}^{\prime \prime}(0)\right|^{2}\left[1-\frac{16 \alpha_{s}}{3 \pi}\right]$

For the $n^{3} D_{1}$ bound states annihilation into $l^{+} l^{-}$have same first order QCD correction factor as of $n^{3} S_{1}$ bound states [114]. The leptonic decay widths are tabulated in Table 6 with and without QCD corrections. We have compared our results with other available experimental as well as theoretical data.

Table 4 The vector decay constant (in $\mathrm{MeV}$ ) of the $S$ wave bottomonium states. $f_{v}$ and $f_{v}(C)$ are present results without and with QCD correction respectively. The Experimental data is taken from [43]

\begin{tabular}{llllllllll}
\hline State & $J^{P C}$ & $f_{V}$ & $f_{V}(C)$ & PDG [43] & [90] & [93] & [92] & [91] & [94] \\
\hline $1^{3} S_{1}$ & $1^{--}$ & 653.44 & 551.53 & $715 \pm 5$ & $649(31)$ & 705.4 & 706 & $498 \pm(20)$ & 831 \\
$2^{3} S_{1}$ & $1^{--}$ & 563.73 & 477.05 & $498 \pm 8$ & $481(39)$ & 554.9 & 547 & $366 \pm(27)$ & 566 \\
$3^{3} S_{1}$ & $1^{--}$ & 507.90 & 430.42 & $430 \pm 4$ & $\ldots$ & 436.8 & 484 & $304 \pm(27)$ & 507 \\
$4^{3} S_{1}$ & $1^{--}$ & 466.60 & 395.80 & $336 \pm 18$ & $\ldots$ & 332.4 & 446 & $259 \pm(22)$ & 481 \\
$5^{3} S_{1}$ & $1^{--}$ & 434.61 & 368.91 & $\ldots$ & $\ldots$ & 286.5 & 419 & $228 \pm(16)$ \\
$6^{3} S_{1}$ & $1^{--}$ & 406.39 & 345.40 & $\ldots$ & $\ldots$ & $\ldots$ & $\ldots$ & $\ldots$ & 458 \\
\hline
\end{tabular}

Table 5 The pseudoscalar decay constant (in $\mathrm{MeV}$ ) of the $S$ wave bottomonium states. $f_{p}$ and $f_{p}(C)$ are present results without and with QCD correction respectively. There is no Experimental data available for comparison. Predictions from Lattice QCD and QCD sum rules are compared

\begin{tabular}{lllllllllc}
\hline State & $J^{P C}$ & $f_{P}$ & $f_{P}(C)$ & {$[95]$} & {$[96]$} & Lattice [98] & [94] & QCD sum rules [99] & [97] \\
\hline $1^{1} S_{0}$ & $0^{-+}$ & 654.81 & 578.21 & 646.025 & 756 & 667 & 834 & $251 \pm 0.072$ \\
$2^{1} S_{0}$ & $0^{-+}$ & 564.59 & 499.48 & 518.803 & 285 & $\ldots$ & 567 & $\ldots$ & 1016.8 \\
$3^{1} S_{0}$ & $0^{-+}$ & 508.53 & 450.35 & 474.954 & 333 & $\ldots$ & 508 & $\ldots$ & 806.6 \\
$4^{1} S_{0}$ & $0^{-+}$ & 467.09 & 413.93 & 449.654 & 40 & $\ldots$ & $\ldots$ & $\ldots$ & $\ldots$ \\
$5^{1} S_{0}$ & $0^{-+}$ & 435.00 & 385.68 & 432.072 & $\ldots$ & $\ldots$ & $\ldots$ & $\ldots$ & $\ldots$ \\
$6^{1} S_{0}$ & $0^{-+}$ & 406.71 & 360.93 & & $\ldots$ & $\ldots$ & $\ldots$ & $\ldots$ & $\ldots$ \\
\hline
\end{tabular}




\subsection{The Di-gamma decay of bottomonium states}

Adopting the case of the para positronium $\left({ }^{1} S_{0}\right)$ state decays to two photons to that of the ${ }^{1} S_{0}$ state of quarkonium with the inclusion of color factor, the expression to compute digamma decay width of pseudoscalar mesons can be written as [74]

$$
\begin{aligned}
\Gamma\left(n^{1} S_{0} \rightarrow \gamma \gamma\right)= & \frac{12 \alpha_{e}^{2} e_{Q}^{4}}{M^{2}\left(n^{1} S_{0}\right)}\left|R_{n l}(0)\right|^{2} \\
& \times\left[1-\frac{\alpha_{s}}{\pi}\left(\frac{20-\pi^{2}}{3}\right)\right]
\end{aligned}
$$

where bracketed quantity is the lowest order QCD correction [82]. In the case of $P$ wave states, decay width depends on the first derivative of the radial wave function at the origin and is given by [82]

$$
\begin{aligned}
\Gamma\left(n^{3} P_{0} \rightarrow \gamma \gamma\right)= & \frac{27 \alpha_{e}^{2} 2^{4}}{M\left(n^{3} P_{0}\right)^{4}} e_{Q}^{4}\left|R_{n l}^{\prime}(0)\right|^{2} \\
& \times\left[1+\frac{\alpha_{s}}{\pi}\left(\frac{\pi^{3}}{3}-\frac{28}{9}\right)\right] \\
\Gamma\left(n^{3} P_{2} \rightarrow \gamma \gamma\right)= & \frac{36 \alpha_{e}^{2} 2^{4}}{5 M\left(n^{3} P_{2}\right)^{4}} e_{Q}^{4}\left|R_{n l}^{\prime}(0)\right|^{2} \\
& \times\left[1-\left(\frac{16 \alpha_{s}}{3 \pi}\right)\right]
\end{aligned}
$$

Table 7 summarises our computed results for the di-gamma decay width of $n^{1} S_{0}$ and $n^{3} P_{0,2}$ states and are compared with other available data.

\subsection{The Di-gluon decay of bottomonium states}

Similar to the di-photon decay, states holding even charge conjugation can decay into di-gluons. Expression used to compute $S$ wave spin-singlet state decaying into two gluons together with the QCD correction is given by [82]

$\Gamma\left(n^{1} S_{0} \rightarrow g g\right)=\frac{2 \alpha_{s}^{2}}{3 m_{Q}^{2}}\left|R_{n l}(0)\right|^{2}\left[1+\frac{4.4 \alpha_{s}}{\pi}\right]$

Conventionally, theoretical expression for di-gluon decay width of the $\chi_{b_{0}}$ and $\chi_{b_{2}}$ states depend on the first derivative of the radial wave function at origin and the formula adopted here for the computation with QCD correction factor can be written as $[82,85,86]$

$\Gamma\left(n^{3} P_{0} \rightarrow g g\right)=\frac{6 \alpha_{s}^{2} 2^{4}}{M\left(n^{3} P_{0}\right)^{4}}\left|R_{n l}^{\prime}(0)\right|^{2}\left[1+\frac{10.0 \alpha_{s}}{\pi}\right]$

$\Gamma\left(n^{3} P_{2} \rightarrow g g\right)=\frac{8 \alpha_{s}^{2} 2^{4}}{5 M\left(n^{3} P_{2}\right)^{4}}\left|R_{n l}^{\prime}(0)\right|^{2}\left[1-\frac{0.1 \alpha_{s}}{\pi}\right]$

The calculated di-gluon decay width with other available theoretical data are listed in Table 8.

\subsection{The Tri-gluon decay of bottomonium states}

The decay width of $S$ wave vector state annihilates into three gluons along with QCD radiative correction is computed using the relation given by $[82,113]$

$$
\begin{aligned}
\Gamma\left(n^{3} S_{1} \rightarrow g g g\right)= & \frac{10 \alpha_{s}^{3} 2^{2}\left(\pi^{2}-9\right)}{81 \pi M\left(n^{3} S_{1}\right)^{2}}\left|R_{n l}(0)\right|^{2} \\
& \times\left[1-\frac{4.9 \alpha_{s}}{\pi}\right]
\end{aligned}
$$

And the state $n^{1} P_{1}$, annihilates to the three gluons is given by $[41,82,113]$

$\Gamma\left(n^{1} P_{1} \rightarrow g g g\right)=\frac{20 \alpha_{s}^{3} 2^{4}}{9 \pi M\left(n^{1} P_{1}\right)^{4}}\left|R_{n l}^{\prime}(0)\right|^{2} \ln \left(m_{Q}\langle r\rangle\right)$

In the case of D-wave, $n^{3} D_{1,3}$ states, the three gluon decay widths are computed using the expression given by [87]

$\Gamma\left(n^{3} D_{1} \rightarrow g g g\right)=\frac{760 \alpha_{s}^{3} 2^{6}}{81 \pi M\left(n^{3} D_{1}\right)^{6}}\left|R_{n l}^{\prime \prime}(0)\right|^{2} \ln \left(4 m_{Q}\langle r\rangle\right)$

$\Gamma\left(n^{3} D_{3} \rightarrow g g g\right)=\frac{40 \alpha_{s}^{3} 2^{6}}{9 \pi M\left(n^{3} D_{3}\right)^{6}}\left|R_{n l}^{\prime \prime}(0)\right|^{2} \ln \left(4 m_{Q}\langle r\rangle\right)$

The computed three gluon decay widths for $S, P$ and $D$ waves are given in Table 9 along with other model predictions.

3.6 Other annihilation channels of vector bottomonium states

Apart from the decays discussed above, there are other processes by which quarkonium states can annihilate. To elaborate specifically, the decay width of mixed strong and electromagnetic annihilation of $n^{3} S_{1}$ states into $\gamma g g$ photon and two gluons is given by [45]

$$
\begin{aligned}
\Gamma\left(n^{3} S_{1} \rightarrow \gamma g g\right)= & \frac{8\left(\pi^{2}-9\right) \alpha \alpha_{s}{ }^{2} e_{Q}{ }^{2} 2^{2}}{9 \pi M\left(n^{3} S_{1}\right)^{2}}\left|R_{n l}(0)\right|^{2} \\
& \times\left[1-\frac{7.4 \alpha_{s}}{\pi}\right],
\end{aligned}
$$

and that of $\gamma \gamma \gamma$ is given by [45]

$$
\begin{aligned}
\Gamma\left(n^{3} S_{1} \rightarrow \gamma \gamma \gamma\right)= & \frac{16\left(\pi^{2}-9\right) \alpha^{3} e_{Q}{ }^{6} 2^{2}}{3 M\left(n^{3} S_{1}\right)^{2}}\left|R_{n l}(0)\right|^{2} \\
& \times\left[1-\frac{12.6 \alpha_{s}}{\pi}\right]
\end{aligned}
$$

Also, $n^{3} P_{1}$ state decay into light flavour mesons with a single gluon, the decay rate is computed using the relation 
Table 6 The di-leptonic decay widths (in $\mathrm{keV}$ ) of the $S$ wave and $D$ wave bottomonium states. $\Gamma_{l^{+} l^{-}}$and $\Gamma_{l^{+} l^{-}}(C)$ are present results without and with QCD correction respectively

\begin{tabular}{|c|c|c|c|c|c|c|c|c|c|c|c|}
\hline State & $J^{P C}$ & $\Gamma_{l^{+} l^{-}}$ & $\Gamma_{l^{+} l^{-}}(C)$ & PDG [43] & {$[100]$} & [41] & {$[101]$} & [93] & [77] & [103] & [102] \\
\hline $1^{3} S_{1}$ & $1^{--}$ & 1.1191 & 0.7700 & $1.34 \pm 0.018$ & 1.33 & 0.71 & 1.20 & 1.30 & 1.809 & 1.3 & 1.60 \\
\hline $2^{3} S_{1}$ & $1^{--}$ & 0.7859 & 0.5442 & $0.612 \pm 0.011$ & 0.62 & 0.37 & 0.52 & 0.76 & 0.797 & 0.5 & 0.64 \\
\hline $3^{3} S_{1}$ & $1^{--}$ & 0.6171 & 0.4288 & $0.443 \pm 0.008$ & 0.48 & 0.27 & 0.33 & 0.45 & 0.618 & $\ldots$ & 0.44 \\
\hline $4^{3} S_{1}$ & $1^{--}$ & 0.5096 & 0.3549 & $0.272 \pm 0.029$ & 0.40 & 0.21 & 0.24 & 0.26 & 0.541 & $\ldots$ & 0.35 \\
\hline $5^{3} S_{1}$ & $1^{--}$ & 0.4350 & 0.3035 & $\ldots$ & $\ldots$ & 0.18 & 0.19 & 0.18 & $\cdots$ & $\ldots$ & 0.29 \\
\hline $6^{3} S_{1}$ & $1^{--}$ & 0.3695 & 0.2586 & $\ldots$ & $\ldots$ & 0.15 & 0.15 & $\ldots$ & $\ldots$ & $\ldots$ & $\ldots$ \\
\hline $1^{3} D_{1}$ & $1^{--}$ & 0.0072 & 0.0050 & $\ldots$ & $\ldots$ & $\ldots$ & $\ldots$ & 0.106 & $\ldots$ & $\ldots$ & $\ldots$ \\
\hline $2^{3} D_{1}$ & $1^{--}$ & 0.0084 & 0.0058 & $\cdots$ & $\ldots$ & $\ldots$ & $\ldots$ & 0.078 & $\ldots$ & $\ldots$ & $\ldots$ \\
\hline $3^{3} D_{1}$ & $1^{--}$ & 0.0085 & 0.0059 & $\cdots$ & $\cdots$ & $\cdots$ & $\cdots$ & 0.051 & $\cdots$ & $\cdots$ & $\cdots$ \\
\hline $4^{3} D_{1}$ & $1^{--}$ & 0.0084 & 0.0058 & $\cdots$ & $\ldots$ & $\cdots$ & $\cdots$ & 0.042 & $\cdots$ & $\cdots$ & $\cdots$ \\
\hline $5^{3} D_{1}$ & $1^{--}$ & 0.0082 & 0.0057 & $\ldots$ & $\ldots$ & $\ldots$ & $\ldots$ & 0.028 & $\ldots$ & $\ldots$ & $\ldots$ \\
\hline $6^{3} D_{1}$ & $1^{--}$ & 0.0079 & 0.0055 & $\cdots$ & $\cdots$ & $\cdots$ & $\cdots$ & $\cdots$ & $\cdots$ & $\cdots$ & $\cdots$ \\
\hline $7^{3} D_{1}$ & $1^{--}$ & 0.0076 & 0.0053 & $\ldots$ & $\ldots$ & $\ldots$ & $\ldots$ & $\ldots$ & $\ldots$ & $\ldots$ & $\ldots$ \\
\hline
\end{tabular}

Table 7 The di-gamma decay widths (in $\mathrm{keV}$ ) of the $S$ wave and $P$ wave bottomonium states. $\Gamma_{\gamma \gamma}$ and $\Gamma_{\gamma \gamma}(C)$ are present results without and with QCD correction respectively. There is no experimental data available for comparison

\begin{tabular}{|c|c|c|c|c|c|c|c|c|c|c|}
\hline State & $J^{P C}$ & $\Gamma_{\gamma \gamma}$ & $\Gamma_{\gamma \gamma}(C)$ & [95] & [41] & [97] & {$[101]$} & [102] & [103] & [1] \\
\hline $1^{1} S_{0}$ & $0^{-+}$ & 0.3782 & 0.3035 & 0.387 & 0.69 & 0.738 & 0.496 & 0.527 & 0.35 & 0.214 \\
\hline $2^{1} S_{0}$ & $0^{-+}$ & 0.2636 & 0.2122 & 0.263 & 0.36 & 0.508 & 0.212 & 0.263 & 0.15 & 0.121 \\
\hline $3^{1} S_{0}$ & $0^{-+}$ & 0.2067 & 0.1668 & 0.229 & 0.27 & 0.426 & 0.135 & 0.172 & 0.10 & 0.906 \\
\hline $4{ }^{1} S_{0}$ & $0^{-+}$ & 0.1705 & 0.1378 & 0.201 & $\ldots$ & $\ldots$ & $\ldots$ & 0.099 & 0.105 & 0.755 \\
\hline $5^{1} S_{0}$ & $0^{-+}$ & 0.1455 & 0.1176 & 0.193 & $\ldots$ & $\ldots$ & $\ldots$ & 0.078 & 0.121 & $\ldots$ \\
\hline $6^{1} S_{0}$ & $0^{-+}$ & 0.1235 & 0.1000 & $\ldots$ & $\ldots$ & $\ldots$ & $\ldots$ & & & $\ldots$ \\
\hline $1^{3} P_{0}$ & $0^{++}$ & 0.0811 & 0.1150 & 0.0196 & 0.12 & $\ldots$ & $\ldots$ & 0.050 & 0.038 & 0.0208 \\
\hline $2^{3} P_{0}$ & $0^{++}$ & 0.0717 & 0.1014 & 0.0195 & 0.14 & $\ldots$ & $\ldots$ & 0.037 & 0.029 & 0.0227 \\
\hline $3^{3} P_{0}$ & $0^{++}$ & 0.0620 & 0.0875 & 0.0194 & 0.15 & $\ldots$ & $\ldots$ & 0.037 & $\ldots$ & $\ldots$ \\
\hline $4^{3} P_{0}$ & $0^{++}$ & 0.0545 & 0.0768 & 0.0192 & $\ldots$ & $\ldots$ & $\ldots$ & $\ldots$ & $\ldots$ & $\ldots$ \\
\hline $5^{3} P_{0}$ & $0^{++}$ & 0.0487 & 0.0686 & $\ldots$ & $\ldots$ & $\ldots$ & $\ldots$ & $\ldots$ & $\ldots$ & $\ldots$ \\
\hline $1^{3} P_{2}$ & $2^{++}$ & 0.0213 & 0.0147 & 0.0052 & 0.00308 & $\ldots$ & $\ldots$ & 0.0066 & 0.008 & 0.0051 \\
\hline $2^{3} P_{2}$ & $2^{++}$ & 0.0189 & 0.0131 & 0.0052 & 0.00384 & $\ldots$ & $\ldots$ & 0.0067 & 0.006 & 0.0062 \\
\hline $3^{3} P_{2}$ & $2^{++}$ & 0.0164 & 0.0114 & 0.0051 & 0.00410 & $\ldots$ & $\ldots$ & 0.0064 & $\ldots$ & $\ldots$ \\
\hline $4^{3} P_{2}$ & $2^{++}$ & 0.0144 & 0.0100 & 0.0051 & $\ldots$ & $\ldots$ & $\ldots$ & $\ldots$ & $\ldots$ & $\ldots$ \\
\hline $5^{3} P_{2}$ & $2^{++}$ & 0.0129 & 0.0090 & $\ldots$ & $\ldots$ & $\ldots$ & $\ldots$ & $\ldots$ & $\ldots$ & $\ldots$ \\
\hline
\end{tabular}

given by [82]

$\Gamma\left(n^{3} P_{1} \rightarrow q \bar{q}+g\right)=\frac{8 \alpha_{s}^{3} n_{f} 2^{4}}{9 \pi M\left(n^{3} P_{1}\right)^{4}}\left|R_{n l}^{\prime}(0)\right|^{2} \ln \left(m_{Q}\langle r\rangle\right)$

The computed results of these decays are summarised in Table 10 along with other available theoretical and experimental data.
3.7 The electromagnetic transition widths of bottomonium states

Bottomonium states possess more compactness in nature due to relatively heavier mass of the bottom quark. In such situation especially dealing with the radiative transition which are governed by an emission or absorption of gamma photon, the wave length of the photon is either larger or comparable to the size of the radiating bottomonium state. So, one expects radiative transition in $b \bar{b}$ dominates. The leading order elec- 
Table 8 The di-gluon decay widths (in $\mathrm{MeV}$ ) of the $S$ wave and $P$ wave bottomonium states. $\Gamma_{g g}$ and $\Gamma_{g g}(C)$ are present results without and with QCD correction respectively. There is no experimental data available for comparison

\begin{tabular}{|c|c|c|c|c|c|c|c|c|}
\hline State & $J^{P C}$ & $\Gamma_{g g}$ & $\Gamma_{g g}(C)$ & [95] & [41] & [105] & [104] & [106] \\
\hline $1^{1} S_{0}$ & $0^{-+}$ & 5.4496 & 6.8520 & 5.448 & 20.18 & 17.945 & 11.49 & 12.46 \\
\hline $2^{1} S_{0}$ & $0^{-+}$ & 4.1775 & 5.2374 & 3.710 & 10.64 & $\ldots$ & 5.16 & $\ldots$ \\
\hline $3^{1} S_{0}$ & $0^{-+}$ & 3.4499 & 4.3182 & 3.229 & 7.94 & $\ldots$ & 3.80 & $\ldots$ \\
\hline $4^{1} S_{0}$ & $0^{-+}$ & 2.9454 & 3.6829 & 2.985 & $\ldots$ & $\ldots$ & $\ldots$ & $\ldots$ \\
\hline $5^{1} S_{0}$ & $0^{-+}$ & 2.5769 & 3.2196 & 2.832 & $\ldots$ & $\ldots$ & $\ldots$ & $\ldots$ \\
\hline $6^{1} S_{0}$ & $0^{-+}$ & 2.2859 & 2.8519 & $\ldots$ & $\ldots$ & $\ldots$ & $\ldots$ & $\ldots$ \\
\hline $1^{3} P_{0}$ & $0^{++}$ & 0.9056 & 1.4297 & 0.276 & 2.00 & 5.250 & 0.96 & 2.15 \\
\hline $2^{3} P_{0}$ & $0^{++}$ & 0.7855 & 1.2358 & 0.275 & 2.37 & $\ldots$ & 0.99 & $\ldots$ \\
\hline $3^{3} P_{0}$ & $0^{++}$ & 0.6713 & 1.0539 & 0.273 & 2.46 & $\cdots$ & $\cdots$ & $\cdots$ \\
\hline $4^{3} P_{0}$ & $0^{++}$ & 0.5853 & 0.9175 & 0.271 & $\ldots$ & $\ldots$ & $\cdots$ & $\cdots$ \\
\hline $5^{3} P_{0}$ & $0^{++}$ & 0.5192 & 0.8127 & $\ldots$ & $\ldots$ & $\ldots$ & $\ldots$ & $\ldots$ \\
\hline $1^{3} P_{2}$ & $2^{++}$ & 0.2384 & 0.2370 & 0.073 & 0.0836 & 0.822 & 0.33 & 0.22 \\
\hline $2^{3} P_{2}$ & $2^{++}$ & 0.2076 & 0.2064 & 0.073 & 0.1042 & $\ldots$ & 0.35 & $\ldots$ \\
\hline $3^{3} P_{2}$ & $2^{++}$ & 0.1778 & 0.1767 & 0.072 & 0.1114 & $\ldots$ & $\cdots$ & $\cdots$ \\
\hline $4^{3} P_{2}$ & $2^{++}$ & 0.1552 & 0.1543 & 0.072 & $\ldots$ & $\ldots$ & $\ldots$ & $\ldots$ \\
\hline $5^{3} P_{2}$ & $2^{++}$ & 0.1378 & 0.1370 & $\ldots$ & $\ldots$ & $\ldots$ & $\ldots$ & $\ldots$ \\
\hline
\end{tabular}

Table 9 The Tri-gluon decay widths (in $\mathrm{MeV}$ ) of the $S, P$ and $D$ wave bottomonium states. $\Gamma_{g g g}$ and $\Gamma_{g g g}(C)$ are present results without and with QCD correction respectively

\begin{tabular}{|c|c|c|c|c|c|}
\hline State & $J^{P C}$ & $\Gamma_{g g g}$ & $\Gamma_{g g g}(C)$ & PDG [43] & [41] \\
\hline $1^{3} S_{1}$ & $1^{--}$ & 0.0400 & 0.0285 & 0.0441 & 0.0416 \\
\hline $2^{3} S_{1}$ & $1^{--}$ & 0.0269 & 0.0193 & 0.0188 & 0.0242 \\
\hline $3^{3} S_{1}$ & $1^{--}$ & 0.0206 & 0.0148 & 0.0072 & 0.01876 \\
\hline $4^{3} S_{1}$ & $1^{--}$ & 0.0168 & 0.0121 & $\ldots$ & $\ldots$ \\
\hline $5^{3} S_{1}$ & $1^{--}$ & 0.0141 & 0.0102 & $\ldots$ & $\ldots$ \\
\hline $6^{3} S_{1}$ & $1^{--}$ & 0.0117 & 0.0085 & $\ldots$ & $\ldots$ \\
\hline $1^{1} P_{1}$ & $1^{+-}$ & 0.0357 & $\ldots$ & $\ldots$ & 0.03526 \\
\hline $2^{1} P_{1}$ & $1^{+-}$ & 0.0346 & $\ldots$ & $\ldots$ & 0.0527 \\
\hline $3^{1} P_{1}$ & $1^{+-}$ & 0.0331 & $\ldots$ & $\ldots$ & 0.0621 \\
\hline $4^{1} P_{1}$ & $1^{+-}$ & 0.0327 & $\ldots$ & $\ldots$ & $\ldots$ \\
\hline $5^{1} P_{1}$ & $1^{+-}$ & 0.0309 & $\ldots$ & $\ldots$ & $\ldots$ \\
\hline $1^{3} D_{1}$ & $1^{--}$ & 0.0106 & $\ldots$ & $\ldots$ & 0.0099 \\
\hline $2^{3} D_{1}$ & $1^{--}$ & 0.0119 & $\ldots$ & $\ldots$ & 0.0096 \\
\hline $3^{3} D_{1}$ & $1^{--}$ & 0.0118 & $\ldots$ & $\ldots$ & $\ldots$ \\
\hline $4^{3} D_{1}$ & $1^{--}$ & 0.0113 & $\ldots$ & $\ldots$ & $\ldots$ \\
\hline $5^{3} D_{1}$ & $1^{--}$ & 0.0108 & $\ldots$ & $\ldots$ & $\ldots$ \\
\hline $1^{3} D_{3}$ & $3^{--}$ & 0.0060 & $\ldots$ & $\ldots$ & 0.00022 \\
\hline $2^{3} D_{3}$ & $3^{--}$ & 0.0056 & $\ldots$ & $\ldots$ & 0.00125 \\
\hline $3^{3} D_{3}$ & $3^{--}$ & 0.0055 & $\ldots$ & $\ldots$ & $\ldots$ \\
\hline $4^{3} D_{3}$ & $3^{--}$ & 0.0053 & $\ldots$ & $\ldots$ & $\ldots$ \\
\hline $5^{3} D_{3}$ & $3^{--}$ & 0.0051 & $\ldots$ & $\ldots$ & $\ldots$ \\
\hline
\end{tabular}


Table 10 Some other annihilation decay widths (in $\mathrm{keV}$ ) of the $S$ and $P$ wave bottomonium states. $\Gamma$ and $\Gamma(C)$ are present results without and with QCD correction respectively

\begin{tabular}{lllll}
\hline Transition & $\Gamma$ & $\Gamma(C)$ & PDG [43] & {$[41]$} \\
\hline $1^{3} S_{1} \rightarrow \gamma \gamma \gamma$ & $4.6775 \times 10^{-4}$ & $1.2312 \times 10^{-4}$ & $\ldots$ & $3.44 \times 10^{-6}$ \\
$2^{3} S_{1} \rightarrow \gamma \gamma \gamma$ & $3.2849 \times 10^{-4}$ & $8.9895 \times 10^{-5}$ & $\ldots$ & $2.00 \times 10^{-6}$ \\
$3^{3} S_{1} \rightarrow \gamma \gamma \gamma$ & $2.5794 \times 10^{-4}$ & $7.2037 \times 10^{-5}$ & $\ldots$ & $1.55 \times 10^{-6}$ \\
$4^{3} S_{1} \rightarrow \gamma \gamma \gamma$ & $2.1298 \times 10^{-4}$ & $6.0336 \times 10^{-5}$ & $\ldots$ & $1.29 \times 10^{-6}$ \\
$5^{3} S_{1} \rightarrow \gamma \gamma \gamma$ & $1.8183 \times 10^{-4}$ & $5.2021 \times 10^{-5}$ & $\ldots$ & $1.10 \times 10^{-6}$ \\
$6^{3} S_{1} \rightarrow \gamma \gamma \gamma$ & $1.5446 \times 10^{-4}$ & $4.4933 \times 10^{-5}$ & $\ldots$ & $9.56 \times 10^{-7}$ \\
$1^{3} S_{1} \rightarrow \gamma g g$ & 1.2730 & 0.7220 & 1.18 & 0.79 \\
$2^{3} S_{1} \rightarrow \gamma g g$ & 0.8689 & 0.4982 & 0.59 & 0.46 \\
$3^{3} S_{1} \rightarrow \gamma g g$ & 0.6718 & 0.3874 & 0.0097 & 0.36 \\
$4^{3} S_{1} \rightarrow \gamma g g$ & 0.5485 & 0.3176 & $\ldots$ & 0.30 \\
$5^{3} S_{1} \rightarrow \gamma g g$ & 0.4646 & 0.2698 & $\ldots$ & 0.25 \\
$6^{3} S_{1} \rightarrow \gamma g g$ & 0.3894 & 0.2272 & $\ldots$ & \\
$1^{3} P_{1} \rightarrow q \bar{q}+g$ & 57.9585 & $\ldots$ & $\ldots$ & $\ldots .22$ \\
$2^{3} P_{1} \rightarrow q \bar{q}+g$ & 55.3966 & $\ldots$ & $\ldots$ & $\ldots$ \\
$3^{3} P_{1} \rightarrow q \bar{q}+g$ & 52.9585 & $\ldots$ & $\ldots$ & $\ldots$ \\
$4^{3} P_{1} \rightarrow q \bar{q}+g$ & 52.4466 & $\ldots$ & $\ldots$ & 1.53 \\
$5^{3} P_{1} \rightarrow q \bar{q}+g$ & 49.5181 & & $\ldots$ &
\end{tabular}

Table 11 The root mean square radii (in fm) for bottomonium states

\begin{tabular}{ll}
\hline State & $\left\langle r^{2}\right\rangle^{\frac{1}{2}}$ \\
\hline $1 S$ & 0.19 \\
$2 S$ & 0.37 \\
$3 S$ & 0.53 \\
$4 S$ & 0.68 \\
$5 S$ & 0.83 \\
1P & 0.27 \\
2P & 0.44 \\
3P & 0.60 \\
4P & 0.75 \\
1D & 0.35 \\
2D & 0.51 \\
3D & 0.67 \\
$4 D$ & 0.82 \\
5D & 0.97 \\
\hline
\end{tabular}

tromagnetic transitions are electric dipole $(E 1)$ and magnetic dipole $(M 1)$ transitions.

The selection rules for electric dipole transition $(E 1)$ are $\Delta l= \pm 1, \Delta s=0$. In contrast, for magnetic dipole transitions $(M 1), \Delta l=0, \Delta s= \pm 1$. Within non relativistic limit, the decay width of the $E 1$ transition from the initial state $n_{i}^{\left(2 s_{i}+1\right)} l_{i J_{i}}$ to final state $n_{f}^{\left(2 s_{f}+1\right)} l_{f_{J_{f}}}$ can be obtained as [2]
$\Gamma(i \stackrel{E 1}{\rightarrow} f+\gamma)=\frac{4 \alpha e_{Q}^{2}}{3}\left(2 J^{\prime}+1\right) S_{i f}^{E} \omega^{3}\left|\mathcal{E}_{i f}\right|^{2} \times \frac{E_{f}}{M_{i}}$

where

$\omega=\frac{M_{i}^{2}-M_{f}^{2}}{2 M_{i}}$

is the photon energy. $S_{i f}^{E}$ is the statistical factor and $\varepsilon_{i f}$ is the overlap integral which can be computed using initial and final state wave functions as

$$
\begin{aligned}
\mathcal{E}_{i f}= & \frac{3}{\omega} \int_{0}^{\infty} R_{n \ell}(r) R_{n^{\prime} \ell^{\prime}}(r) r^{2} d r \\
& \times\left[\frac{\omega r}{2} j_{0}\left(\frac{\omega r}{2}\right)-j_{1}\left(\frac{\omega r}{2}\right)\right]
\end{aligned}
$$

and

$S_{i f}^{E}=\max \left(\ell, \ell^{\prime}\right)\left\{\begin{array}{lll}J & 1 & J^{\prime} \\ \ell^{\prime} & s & \ell\end{array}\right\}^{2}$

The formula used for the $(M 1)$ transition from initial state to final state for the quarkonium system can be computed as $[88,89]$

$$
\begin{aligned}
\Gamma(i \stackrel{M 1}{\longrightarrow} f+\gamma)= & \frac{4 \alpha e_{Q}^{2}}{3 m_{Q^{2}}}\left(2 J^{\prime}+1\right) S_{i f}^{M} \\
& \times \omega^{3}\left|\left\langle f\left|j_{0}\left(\frac{\omega r}{2}\right)\right| i\right\rangle\right|^{2} \times \frac{E_{f}}{M_{i}}
\end{aligned}
$$

The statistical factor for the $(M 1)$ transition is given as $S_{i f}^{M}=6(2 s+1)\left(2 s^{\prime}+1\right)$ 


$$
\left\{\begin{array}{lll}
J & 1 & J^{\prime} \\
s^{\prime} & l & s
\end{array}\right\}^{2}\left\{\begin{array}{ccc}
1 & \frac{1}{2} & \frac{1}{2} \\
\frac{1}{2} & s^{\prime} & s
\end{array}\right\}^{2}
$$

It is to be noted that the term $E_{f} / M_{i}$ is acting as the relativistic correction factor to the radiative transition width where $E_{f}$ is the energy of the final state and $M_{i}$ being the mass of the initial state. The criteria for validity of these transitions to take place within long wave length approximation (LWLA) is specified as $\omega\left(2\left\langle r^{2}\right\rangle_{f}^{\frac{1}{2}}<1\right.$ [116]. Table 12 shows calculated values for $\omega\left(2\left\langle r^{2}\right\rangle_{f}^{\frac{1}{2}}\right.$ using our predicted masses of the bottomonium states. Possible $E 1$ and $M 1$ transitions including relativistic correction factor and satisfying the LWLA are tabulated in Tables 13 and 14 along with the available theoretical and experimental data for comparison.

\section{Results and discussion}

The spectroscopic masses of S, P and D waves of the bottomonia are computed based on the instanton induced potential in the non-relativistic frame work with the addition of stronger confinement effects. The present results are compared with available experimental as well as with other model predictions in Table 2 for $\mathrm{S}$ wave masses and in Table 3 for $\mathrm{P}$ wave and $\mathrm{D}$ wave masses. Our results are found to be in very good agreement with the experimental values of the respective states. Our estimation for the $1^{3} S_{1}$ is $9460.75 \mathrm{MeV}$ which is in excellent agreement with the PDG listed mass of $1^{3} S_{1}(9460.30 \pm 0.26) \mathrm{MeV}$. For the case of spin singlet pseudoscalar state $1^{1} S_{0}$ our finding is 9412.22 MeV which is roughly $13 \mathrm{MeV}$ higher than PDG listed mass $(9398.7 \pm 2.0 \mathrm{MeV})$. The mass splitting of $1 \mathrm{~S}$ state $\left(1^{3} S_{1}-1^{1} S_{0}\right)$ is found to be $48 \mathrm{MeV}$ which is relatively consistent with the PDG listed mass split for $1 \mathrm{~S}$ bottomonium $(62.3 \pm 3.2 \mathrm{MeV})$. We observe that the $\mathrm{S}$ wave mass predictions upto the $4 S$ states are very close to the experimental values (PDG average). Below the $B \bar{B}$ threshold, the spectrum of the bottomonium is well understood. The vector states, $n^{3} S_{1}$ for $n=1,2,3,4$ are well established states. But there exist discrepancy pertaining to the true identification of $\Upsilon(10,860)$ and $\Upsilon(11,020)$ states. Their status as pure $5^{3} S_{1}$ and $6^{3} S_{1}$ of bottomonium states is not clear. It is also evident from the fact that the leptonic decay width $(0.31 \pm 0.07 \mathrm{keV})$ of $\Upsilon(10,860)$ is higher than that of $\Upsilon(4 S)$ $(0.272 \pm 0.029 \mathrm{keV})$ state [43]. The disparity is understood by treating such state of quarkonia as admixture of the $S$ state with the nearby $D$ state. Therefore, identification of these states beyond $4 S$ with $J^{P C}=1^{--}$of quarkonia necessitates the considerations of $S$ wave and $D$ wave admixtures.

Such mixed state $R_{n J}$ in terms of the mixing angle $\theta$ can be represented as

$R_{n J}(0)=\cos \theta R_{n S}(0)-\sin \theta R_{n^{\prime} D}(0)$
Table 12 Photon energy $\omega$ (in $\mathrm{MeV}$ ) calculated from the predicted masses of the states and the LWLA validity factor $\omega\left(2\left\langle r^{2}\right\rangle_{f}^{\frac{1}{2}}\right.$ for (E1) and (M1) transitions

\begin{tabular}{|c|c|c|}
\hline$i \rightarrow f+\gamma$ & $\omega$ & $\omega\left(2\left\langle r^{2}\right\rangle_{f}^{\frac{1}{2}}\right.$ \\
\hline $1^{3} P_{2} \rightarrow 1^{3} S_{1}+\gamma$ & 411.69 & 0.7928 \\
\hline $1^{3} P_{1} \rightarrow 1^{3} S_{1}+\gamma$ & 405.13 & 0.7801 \\
\hline $1^{3} P_{0} \rightarrow 1^{3} S_{1}+\gamma$ & 381.18 & 0.7340 \\
\hline $1^{1} P_{1} \rightarrow 1^{1} S_{0}+\gamma$ & 451.51 & 0.8695 \\
\hline $1^{3} D_{1} \rightarrow 1^{3} P_{0}+\gamma$ & 291.07 & 0.7965 \\
\hline $1^{3} D_{1} \rightarrow 1^{3} P_{1}+\gamma$ & 269.83 & 0.7384 \\
\hline $1^{3} D_{1} \rightarrow 1^{3} P_{2}+\gamma$ & 260.16 & 0.7119 \\
\hline $1^{3} D_{2} \rightarrow 1^{3} P_{1}+\gamma$ & 277.51 & 0.7594 \\
\hline $1^{3} D_{2} \rightarrow 1^{3} P_{2}+\gamma$ & 267.85 & 0.7329 \\
\hline $1^{3} D_{3} \rightarrow 1^{3} P_{2}+\gamma$ & 273.13 & 0.7474 \\
\hline $1^{1} D_{2} \rightarrow 1^{1} P_{1}+\gamma$ & 275.40 & 0.7536 \\
\hline $2^{3} S_{1} \rightarrow 1^{3} P_{0}+\gamma$ & 175.05 & 0.4790 \\
\hline $2^{3} S_{1} \rightarrow 1^{3} P_{1}+\gamma$ & 153.55 & 0.4202 \\
\hline $2^{3} S_{1} \rightarrow 1^{3} P_{2}+\gamma$ & 143.77 & 0.3934 \\
\hline $2^{1} S_{0} \rightarrow 1^{1} P_{1}+\gamma$ & 120.18 & 0.3289 \\
\hline $2^{3} P_{2} \rightarrow 2^{3} S_{1}+\gamma$ & 245.54 & 1.0950 \\
\hline $2^{3} P_{1} \rightarrow 2^{3} S_{1}+\gamma$ & 238.79 & 1.0649 \\
\hline $2^{3} P_{0} \rightarrow 2^{3} S_{1}+\gamma$ & 223.82 & 1.0001 \\
\hline $2^{1} P_{1} \rightarrow 2^{1} S_{0}+\gamma$ & 270.85 & 1.2078 \\
\hline $2^{3} P_{2} \rightarrow 1^{3} S_{1}+\gamma$ & 781.77 & 1.5054 \\
\hline $2^{3} P_{1} \rightarrow 1^{3} S_{1}+\gamma$ & 775.38 & 1.4931 \\
\hline $2^{3} P_{0} \rightarrow 1^{3} S_{1}+\gamma$ & 761.21 & 1.4659 \\
\hline $2^{1} P_{1} \rightarrow 1^{1} S_{0}+\gamma$ & 821.95 & 1.5828 \\
\hline $3^{3} S_{1} \rightarrow 2^{3} P_{0}+\gamma$ & 111.50 & 0.4972 \\
\hline $3^{3} S_{1} \rightarrow 2^{3} P_{1}+\gamma$ & 96.33 & 0.4296 \\
\hline $3^{3} S_{1} \rightarrow 2^{3} P_{2}+\gamma$ & 89.49 & 0.3990 \\
\hline $3^{1} S_{0} \rightarrow 2^{1} P_{1}+\gamma$ & 68.76 & 0.3066 \\
\hline $3^{3} S_{1} \rightarrow 1^{3} P_{0}+\gamma$ & 502.24 & 1.3744 \\
\hline $3^{3} S_{1} \rightarrow 1^{3} P_{1}+\gamma$ & 481.44 & 1.3175 \\
\hline $3^{3} S_{1} \rightarrow 1^{3} P_{2}+\gamma$ & 471.98 & 1.2916 \\
\hline $3^{1} S_{0} \rightarrow 1^{1} P_{1}+\gamma$ & 454.00 & 1.2424 \\
\hline $1^{3} S_{1} \rightarrow 1^{1} S_{0}+\gamma$ & 18.39 & 0.0932 \\
\hline $1^{3} P_{2} \rightarrow 1^{1} P_{1}+\gamma$ & 3.69 & 0.0187 \\
\hline $1^{1} P_{1} \rightarrow 1^{3} P_{1}+\gamma$ & 1.66 & 0.0084 \\
\hline $1^{1} P_{1} \rightarrow 1^{3} P_{0}+\gamma$ & 13.45 & 0.0681 \\
\hline $2^{3} S_{1} \rightarrow 2^{1} S_{0}+\gamma$ & 22.71 & 0.1151 \\
\hline $3^{3} S_{1} \rightarrow 3^{1} S_{0}+\gamma$ & 27.15 & 0.1376 \\
\hline
\end{tabular}

The mixing angle $\theta$ is determined in terms of the $S$ wave mass and the nearby $D$ wave mass as

$M_{n J}=|a|^{2} M_{n S}+\left(1-|a|^{2}\right) M_{n^{\prime} D}$ 
Table 13 The E1 transition decay widhts (in $\mathrm{keV}$ ) of bottomonium states. $\Gamma_{E 1}$ and $\Gamma_{E 1}(R)$ are present results without and with relativistic correction factor respectively. Experimental data is taken from [43]

\begin{tabular}{|c|c|c|c|c|c|c|c|c|}
\hline Initial & Final & $\Gamma_{E 1}$ & $\Gamma_{E 1}(C)$ & PDG [43] & [95] & [109] & [41] & [42] \\
\hline $1^{3} P_{2}$ & $1^{3} S_{1}$ & 15.7013 & 15.0471 & $\ldots$ & 57.530 & 31.8 & 39.15 & 32.8 \\
\hline $1^{3} P_{1}$ & $1^{3} S_{1}$ & 14.6574 & 14.0602 & $\ldots$ & 54.927 & 31.9 & 35.66 & 29.5 \\
\hline $1^{3} P_{0}$ & $1^{3} S_{1}$ & 12.5171 & 12.0327 & $\ldots$ & 49.530 & 27.5 & 28.07 & 23.8 \\
\hline $1^{1} P_{1}$ & $1^{1} S_{0}$ & 19.4593 & 18.5864 & $\ldots$ & 72.094 & 35.8 & 43.66 & 35.7 \\
\hline $1^{3} D_{1}$ & $1^{3} P_{0}$ & 4.4757 & 4.3472 & $\ldots$ & 9.670 & 19.8 & 20.98 & 16.5 \\
\hline $1^{3} D_{1}$ & $1^{3} P_{1}$ & 2.6836 & 2.6123 & $\ldots$ & 6.313 & 13.3 & 12.29 & 9.7 \\
\hline $1^{3} D_{1}$ & $1^{3} P_{2}$ & 0.1606 & 0.1564 & $\ldots$ & 0.394 & 1.02 & 0.65 & 0.56 \\
\hline $1^{3} D_{2}$ & $1^{3} P_{1}$ & 5.2421 & 5.0988 & $\ldots$ & 11.489 & 21.8 & 21.95 & 19.2 \\
\hline $1^{3} D_{2}$ & $1^{3} P_{2}$ & 1.5736 & 1.5321 & $\ldots$ & 3.583 & 7.23 & 6.23 & 5.6 \\
\hline $1^{3} D_{3}$ & $1^{3} P_{2}$ & 6.6766 & 6.4976 & $\ldots$ & 14.013 & 32.1 & 24.74 & 24.3 \\
\hline $1^{1} D_{2}$ & $1^{1} P_{1}$ & 6.8415 & 6.6567 & $\ldots$ & 14.821 & 30.3 & 17.23 & 24.9 \\
\hline $2^{3} S_{1}$ & $1^{3} P_{0}$ & 0.3021 & 0.2968 & $1.22 \pm 0.11$ & 2.377 & 1.09 & 1.09 & 0.91 \\
\hline $2^{3} S_{1}$ & $1^{3} P_{1}$ & 0.6140 & 0.6045 & $2.21 \pm 0.19$ & 5.689 & 2.17 & 1.84 & 1.63 \\
\hline $2^{3} S_{1}$ & $1^{3} P_{2}$ & 0.8412 & 0.8291 & $2.29 \pm 0.20$ & 8.486 & 2.62 & 2.08 & 1.88 \\
\hline $2^{1} S_{0}$ & $1^{1} P_{1}$ & 0.8877 & 0.8770 & $\ldots$ & 10.181 & 3.41 & 2.85 & 2.48 \\
\hline $3^{3} S_{1}$ & $2^{3} P_{0}$ & 0.2151 & 0.2128 & $1.20 \pm 0.12$ & 3.330 & 1.21 & 1.21 & 1.03 \\
\hline $3^{3} S_{1}$ & $2^{3} P_{1}$ & 0.4175 & 0.4137 & $2.56 \pm 0.26$ & 7.936 & 2.61 & 2.13 & 1.91 \\
\hline $3^{3} S_{1}$ & $2^{3} P_{2}$ & 0.5585 & 0.5536 & $2.66 \pm 0.27$ & 11.447 & 3.16 & 2.56 & 2.30 \\
\hline $3^{1} S_{0}$ & $2^{1} P_{1}$ & 0.4586 & 0.4555 & $\ldots$ & 13.981 & 4.25 & 2.60 & 2.96 \\
\hline
\end{tabular}

Table 14 The M1 transition decay widhts (in eV) of bottomonium states. $\Gamma_{M 1}$ and $\Gamma_{M 1}(C)$ are present results without and with relativistic correction factor respectively. No Experimental data is available comparison

\begin{tabular}{llllllll}
\hline Initial & Final & $\Gamma_{M 1}$ & $\Gamma_{M 1}(C)$ & [95] & [109] & [41] & [42] \\
\hline $1^{3} S_{1}$ & $1^{1} S_{0}$ & 3.7986 & 3.7827 & 37.668 & 10 & 9.34 \\
$1^{3} P_{2}$ & $1^{1} P_{1}$ & 0.0197 & 0.0197 & $\ldots$ & 0.095 & 0.089 \\
$1^{1} P_{1}$ & $1^{3} P_{1}$ & 0.0018 & 0.0018 & $\ldots$ & 0.0094 & 0.0115 \\
$1^{1} P_{1}$ & $1^{3} P_{0}$ & 0.3191 & 0.3183 & $\ldots$ & 0.90 & 0.86 \\
$2^{3} S_{1}$ & $2^{1} S_{0}$ & 1.7856 & 1.7801 & 5.619 & 0.59 & 0.59 \\
$3^{3} S_{1}$ & $3^{1} S_{0}$ & 1.0341 & 1.0315 & 2.849 & 3.9 & 0.096 \\
\hline
\end{tabular}

Table $15 S-D$ mixing parameters for $\Upsilon(10,860)$ and $\Upsilon(11,020)$ states and their predicted leptonic decay widths

\begin{tabular}{|c|c|c|c|c|}
\hline Exp. state & $\begin{array}{l}\text { Mixed state } \\
\text { configuration }\end{array}$ & $\begin{array}{l}\text { Mixing angle } \\
\theta\end{array}$ & $\begin{array}{l}\text { Mixed state } \\
\text { di-leptonic width (in } \mathrm{keV} \text { ) }\end{array}$ & $\begin{array}{l}\text { Exp. decay width } \\
\text { (in } \mathrm{keV} \text { ) }\end{array}$ \\
\hline $10,885.2$ & $5^{3} S_{1}$ and $4^{3} D_{1}$ & Not possible & $\ldots$ & $0.31 \pm 0.07$ \\
\hline $10,885.2$ & $5^{3} S_{1}$ and $5^{3} D_{1}$ & $53.79^{\circ}$ & 0.15 & $0.31 \pm 0.07$ \\
\hline $10,885.2$ & $5^{3} S_{1}$ and $6^{3} D_{1}$ & $39.98^{\circ}$ & 0.25 & $0.31 \pm 0.07$ \\
\hline $11,000.0$ & $6^{3} S_{1}$ and $5^{3} D_{1}$ & $51.69^{\circ}$ & 0.14 & $0.13 \pm 0.03$ \\
\hline $11,000.0$ & $6^{3} S_{1}$ and $6^{3} D_{1}$ & Not possible & $\ldots$ & $0.13 \pm 0.03$ \\
\hline $11,000.0$ & $6^{3} S_{1}$ and $7^{3} D_{1}$ & Not possible & $\ldots$ & $0.13 \pm 0.03$ \\
\hline
\end{tabular}


Table 16 Spin average masses and mass splittings of bottomonium states compared with PDG [43] collected data

\begin{tabular}{lll}
\hline State & Mass Present & Mass $_{\text {Exp }}[43]$ \\
\hline $1 \bar{S}$ & 9448.61 & 9444.97 \\
$2 \bar{S}$ & 10018.53 & 10017.19 \\
$1 \bar{P}-1 \bar{S}$ & 425.95 & 454.9 \\
$1^{3} S_{1}-1^{1} S_{0}$ & 48.53 & $62.3 \pm 3.2$ \\
$2^{3} S_{1}-2^{1} S_{0}$ & 30.74 & $24.3 \pm 3.5_{-1.9}^{+2.8}$ \\
$3^{3} S_{1}-2^{3} S_{1}$ & 338.43 & $331.50 \pm 0.02 \pm 0.13$ \\
\hline
\end{tabular}

Here, $|a|^{2}=\cos ^{2} \theta$. The value of $|a|^{2}$ gives the probability of being in the $M_{n S}$ state while $\left(1-|a|^{2}\right)$ gives the probability of being in the $M_{n^{\prime} D}$ state with mixing angle $\theta$. Table 15 shows possible configuration and the corresponding mixing angles for $\Upsilon(10,860)$ and $\Upsilon(11,020)$ states. For some cases mixing probability is greater than one, they are listed as "not possible" mixing configuration. Following this, $\Upsilon(10,860)$ with a mass of $10,885.2 \mathrm{MeV}$ as reported by PDG is found to be the admixture of $5^{3} S_{1}$ and $6^{3} D_{1}$ states with the mixing angle $\theta=39.98^{\circ}$. And the di-leptonic decay width of this mixed state is estimated as $0.25 \mathrm{keV}$ which is very close to the PDG reported value of $0.31 \pm 0.07 \mathrm{keV}$ rather than the predicted $0.43 \mathrm{keV}$ of pure $5^{3} S_{1}$ state. Similarly, $\Upsilon(11,020)$ of mass $11,000.0 \mathrm{MeV}$ listed by PDG is found to be the admixture of $6^{3} S_{1}$ and $5^{3} D_{1}$ with mixing angle $\theta=51.69^{\circ}$ and di-leptonic decay of the mixed state is obtained as $0.14 \mathrm{keV}$ which is in excellent agreement with the PDG listed value of $0.13 \pm 0.03 \mathrm{keV}$. Fine agreements are also seen in the case of $\mathrm{P}$ wave states and the $\mathrm{D}$ waves with respect to the existing experimental values.

In the Table 16 we have compared the spin average masses and various mass splitting with PDG reported values. The spin average masses of the low-lying states $1 \bar{S}$ from present study is $9448.61 \mathrm{MeV}$ which is very close to $9444.97 \mathrm{MeV}$ as observed from PDG listed masses of $1^{3} S_{1}$ and $1^{1} S_{0}$. Considering $2^{1} S_{0}$ mass of $9999.0 \pm 3.5 \mathrm{MeV}$ predicted by Belle Collaboration and its $2^{3} S_{1}$ mass listed in PDG, we get the $2 \bar{S}$ mass as $10,018.53 \mathrm{MeV}$ which is very close to the predicted $2 \bar{S}$ mass of $10,017.19 \mathrm{MeV}$. Similarly, the centroid masses of the low-lying P-states listed in Table 17 are also in good accord with the values deduced from experimental values.

It is found that the modification incorporated to the instanton induced potential to make it a smoothly varying function of $r$ as well as introducing a strong confinement part has led to the successful predictions of the masses of various states as well as the right amount of hyperfine splitting between the ${ }^{3} S_{1}$ and ${ }^{1} S_{0}$ states and the $P$ and $D$ wave splitting. It has also been observed that by the subtraction of $61.81 \mathrm{MeV}$ from Eq. (2) (short range part of potential) instead of adding to the long range part of potential though removes the discontinuity at $r=0.36 \mathrm{fm}$ but the predictions are not satisfactory as compared to the experimental values even after refitting of the parameters. It can be seen from the Table 11 for the root mean square radii, the rms radii of $1 S, 2 S$ and $1 P$ are nearer to $0.36 \mathrm{fm}$. So, this short range part of the potential though is small but not negligible for these low lying states. It is to be noticed that even though the contribution from short range part of the potential (Eq. (2)) is small compared to other components of the potential, their contributions to the hyperfine spilitting through the variational parameter $\mu$ is not negligible. For instance, if we include only the long range and confinement part in our calculations then in that case, the hyperfine contribution is only $8 \mathrm{MeV}$ which is very much lower than the experimental hyperfine mass splitting of $62.3 \pm 3.2 \mathrm{MeV}$ between $\Upsilon(1 S)$ and $\eta_{b}(1 S)$ states. So, short range part of the potential is crucial for the prediction of spin dependent contributions. Thus, the full potential (short range plus long range plus confinement) part is important for the determination of the wave functions of the hadronic states. These wave functions are crucial for the predictions of the hyperfine, fine structure as well as the decay properties of the mesonic states.

As far as the identification of the excited bottomonion states are concerned, the masses, decay constant, di gamma, di leptonic decay width are primarily important. Comparing the results of the vector decay constants by our formalism with the experimental result of PDG [43] we found that our results are fairly in good agreement. For the orbitally excited states our results with the radiative corrections are in very good agreement with PDG [43] reported values. For instance, the vector decay constant of $3 \mathrm{~S}$ state, our predicted value of

Table 17 The theoretical masses of the ground and few excited states of $\mathrm{P}$ - wave states are compared with the spin-average centroid masses of the corresponding experimental data reported by PDG [43]

\begin{tabular}{lllll}
\hline State & Mass $\left(n^{1} P_{1}\right)_{\text {Present }}$ & Mass $\left(n^{1} P_{1}\right)_{E x p}[43]$ & Mass $\left(n^{3} P_{J}\right)_{\text {Present }}$ & Mass $\left(n^{3} P_{J}\right)_{E x p}[43]$ \\
\hline 1P & 9874.56 & $9899.3 \pm 0.8$ & 9874.56 & $9899.87 \pm 0.27$ \\
2P & $10,270.00$ & $10,259.8 \pm 1.2$ & $10,270.00$ & $10,260.20 \pm 0.36$ \\
3P & $10,526.50$ & $\ldots$ & $10,526.50$ & $10,534 \pm 9$ \\
& & & & $10,551 \pm 1[117]$ \\
& & & $10,530 \pm 4[118]$ \\
\hline
\end{tabular}


$430.42 \mathrm{MeV}$ is very close to the value reported by PDG [43] $(430 \pm 4 \mathrm{MeV})$. This indicates that radiative corrections play an important role in the decay mechanism.

In the case of pseudoscalar decay constant, due to the unavailability of PDG data we have compared our data set with the results of other theoretical model [95], Lattice QCD [98] and QCD sum rules [99]. We found our results in good agreement with the Cornell potential predictions [95]. Also in the case of Lattice QCD, the pseudosalar decay constant for $1^{1} S_{0}$ state is $(667 \mathrm{MeV})$ which is in close agreement with our prediction $(654.81 \mathrm{MeV})$. One can see from the Table 5 that predictions from QCD sum rule [99] is roughly three times lower than all other estimations for $1^{1} S_{0}$ state. Also, the results in the case of [97] (potential model study) are considerably higher than other estimations of the pseudoscalar decay constant. It is important to note that both the vector and the pseudoscalar decay constants rely upon the numerical value of the square of the wave function at the origin, so the choice of the potential and parameters may affect on the predictions. We have also computed the Di-lepton, Di-gamma and Di-gluon decay widths and results are summarized in Table 6, 7 and 8 . Our results are compared with the respective values reported in PDG [43]. In the case of di-leptonic decay of the $n^{3} S_{1}$ it is observed that the radiative corrections are important for higher $(n>2)$ radial excited states. The results predicted by [100] (Relativistic potential model) are much closer to our predictions with radiative corrections. Besides that our predicted results with radiative correction are also in a close agreement with [102] (Screened potential model). In general our results are comparable with other predictions. We have been able to compute the $n^{3} D_{1}$ annihilation into electron positron pair but we don't find more estimations in literature for its comparison.

The di-gamma decay widths are consistent with the outcomes of Cornell potential model [95] for pseudoscalar $n^{1} S_{0}$ states. It is found that the predictions reported by [97] are almost two times higher than all the other reported values. For the $n^{3} P_{0}$ states annihilation into $\gamma \gamma$, our results are comparable with other predictions. Our predictions are found to be higher than other results in the case of $n^{3} P_{2}$ states.

For $n^{3} S_{0}$ decays into $g g$ our estimated results without radiative correction are consistent with [95] and considerably lower than that from the [41,104-106]. For $n^{3} P_{0}$ states, our computed results agree well with the [104] without QCD correction while they are in accordance with results of [41,106] with radiative correction. For these states estimations of [95] are comparable with our predictions. For the $n^{3} P_{2}$ state, our predictions are in line with that of [106] and [104] . For the S wave vector state decaying into $g g g$ our results are in agreement with the decay width listed by PDG [43]. Also they are comparable with [41] especially for $1^{3} S_{1}$ state where our prediction is $0.040 \mathrm{MeV}$ without radiative correction while their prediction is $0.041 \mathrm{MeV}$. For the $n^{1} P_{1}, n^{3} D_{1}$ and $1^{3} D_{3}$ we do not include the radiative correction because such corrections are very small. Interestingly, for $1^{1} P_{1}$ stat, our predicted tri-gluon decay width is $0.035 \mathrm{MeV}$ which is the same as reported by [41].

Table 10 summaries some of the other annihilation decay of the $n^{3} S_{1}$ into $\gamma \gamma \gamma$ and $\gamma g g$. As for the $\gamma \gamma \gamma$ is concerned, we find that present results are considerably higher than [41]. For $\gamma g g$ it is again consistent with the PDG [43] and [41]. The radiatively corrected results are almost matching with that of [41]. Results for $n^{3} P_{1} \rightarrow q \bar{q}+g$ are few keV lower than [41] but still comparable. For all these decays one has to wait for the experimental confirmation. In general, We can conclude that our instanton potential predictions and those from the constituent quark model predictions [41] are in a good agreement for the $g g g$ as well as $\gamma g g$ decays.

Table 12 shows the photon energy computed using predicted masses of the bottomonia states and the corresponding long wavelength approximation (LWLA) validity factor for various electromagnetic transitions. $\omega\left(2\left\langle r^{2}\right\rangle_{f}^{\frac{1}{2}}\right.$. From the table one can find that for transitions $2 P \rightarrow 1 S, 2 P \rightarrow 2 S$ and $3 S \rightarrow 1 P$ the value of $\omega\left(2\left\langle r^{2}\right\rangle_{f}^{\frac{1}{2}}\right.$ is greater than 1 . So, for these transition long wavelength approximation is not applicable and we have not computed their transition widths here. Very recently, Bruschini and Gonzalez [116] worked on the radiative decays in bottomonium beyond the long wavelength approximation and corresponding study are underway. We present some of the allowed electric dipole transitions (E1) and magnetic dipole transitions (M1) as per LWLA in Tables 13 and 14 respectively. Our predicted transition widths are compared with the available other predictions and experimental values wherever it is available. Looking to the results, we find that for $1 P \rightarrow 1 S$ results are much lower than that reported in [95]. And similarly we find that our results deviates from [95] for every transitions except from $1 D \rightarrow 1 P$ where the predictions do agree. On the experimental side, transition width from $1 P \rightarrow 1 S, 1 D \rightarrow 1 P$ is not yet listed in PDG. For the magnetic dipole transitions our predictions are more or less comparable with other data set. The discrepancies in the theoretical predictions may vary from one model to the other due to the choice of potential which plays an important role in the predictions of mass which in turn creates differences in phase space which affects the transition widths. Also sometimes it is a choice of different wave function which effects the predictions of the transition widths. To improve the results one need to consider either the numerical solutions or the perturbative effects on the wave functions for the computation of the electromagnetic transition form factors. Also, instanton vacuum potential plays a vital role in obtaining mass spectroscopy and other relavant properties of bottomonium. Present study re-iterates the importance of the addition of confinement in heavy sector while employing 
instanton effects as highlighted by Schäfer and Shuryak in their review paper [58].

Finally, we hope that our predicted results using instanton effects on heavy quarks will be useful in the identification of new quarkonium states that will be observed in future experiments.

Acknowledgements We acknowledge the partial support from DSTSERB, India through the major research project: (SERB/F/8749/201516).

Data Availability Statement This manuscript has no associated data or the data will not be deposited. [Authors' comment: All data generated or analyzed during this study are included in this published article and the paper has no associated data since it is theoretical work.]

Open Access This article is licensed under a Creative Commons Attribution 4.0 International License, which permits use, sharing, adaptation, distribution and reproduction in any medium or format, as long as you give appropriate credit to the original author(s) and the source, provide a link to the Creative Commons licence, and indicate if changes were made. The images or other third party material in this article are included in the article's Creative Commons licence, unless indicated otherwise in a credit line to the material. If material is not included in the article's Creative Commons licence and your intended use is not permitted by statutory regulation or exceeds the permitted use, you will need to obtain permission directly from the copyright holder. To view a copy of this licence, visit http://creativecomm ons.org/licenses/by/4.0/.

Funded by SCOAP ${ }^{3}$.

\section{References}

1. S. Godfrey, N. Isgur, Phys. Rev. D 32, 189 (1985)

2. E. Eichten, K. Gottfried, T. Kinoshita, K.D. Lane, T.M. Yan, Phys. Rev. D 17, 3090 (1978)

3. A. Garmash, EPJ Web Conf. 96, 01014 (2015)

4. E. Eichten, S. Godfrey, H. Mahlke, J.L. Rosner, Rev. Mod. Phys. 80, 1161 (2008)

5. S. Herb et al., Phys. Rev. Lett. 39, 252 (1977)

6. W.R. Innes et al., Phys. Rev. Lett. 39, 1240 (1977)

7. K. Han et al., Phys. Rev. Lett. 49, 1612 (1982)

8. G. Eigen et al., Phys. Rev. Lett. 49, 1616 (1982)

9. C. Klopfenstein et al., Phys. Rev. Lett. 51, 160 (1983)

10. F. Pauss et al., Phys. Lett. B 130, 439 (1983)

11. D. Lovelock et al., Phys. Rev. Lett. 54, 377 (1985)

12. D. Besson et al. (CLEO), Phys. Rev. Lett. 54, 381 (1985)

13. B. Aubert et al. (BABAR), Phys. Rev. Lett. 101, 071801 (2008)

14. G. Bonvicini et al. (CLEO), Phys. Rev. D 81, 031104 (2010)

15. R. Mizuk et al. (Belle), Phys. Rev. Lett. 109, 232002 (2012)

16. J. Lees et al. (BABAR), Phys. Rev. D 84, 072002 (2011)

17. S. Dobbs, Z. Metreveli, K. Seth, A. Tomaradze, T. Xiao, Phys. Rev. Lett. 109, 082001 (2012)

18. S. Sandilya et al. (Belle), Phys. Rev. Lett. 111, 112001 (2013)

19. G. Bonvicini et al. (CLEO), Phys. Rev. D. 70, 032001 (2004)

20. P. del Amo Sanchez et al. (BABAR), Phys. Rev. D. 82, 111102 (2010)

21. J. Lees et al. (BABAR), Phys. Rev. D 84, 091101 (2011)

22. R. Aaij et al. (LHCb), J. High Energy Phys. 10, 005 (2014)

23. A. Bondar et al. (Belle), Phys. Rev. Lett. 108, 122001 (2012)

24. T. Guo, L. Cao, M. Zhou, H. Chen, arXiv:1106.2284
25. I. Danilkin, V. Orlovsky, Y. Simonov, Phys. Rev. D 85, 034012 (2012)

26. C. Hughes, R.J. Downdall, C.T.H. Davies, R.R. Horgan, G. von Hippel, M. Wingate, Phys. Rev. D 84, 094501 (2015)

27. D. Becirevi, M. Kruse, F. Sanfilippo, J. High Energy Phys. 05, 014 (2011)

28. R. Lewis, R.M. Woloshyn, Phys. Rev. D 84, 094501 (2011)

29. M. Baker, A.A. Penin, D. Seidel, N. Zerf, Phys. Rev. D 92, 054502 (2015)

30. N. Brambilla, Y. Jia, A. Vairo, Phys. Rev. D 73, 054005 (2006)

31. N. Brambilla, P. Pietrulewicz, A. Vairo, Phys. Rev. D 85, 094005 (2012)

32. A. Pineda, J. Segovia, Phys. Rev. D 87, 074024 (2013)

33. H.M. Choi, Phys. Rev. D 75, 073016 (2007)

34. H.W. Ke, X.Q. Li, Z.T. Wei, X. Liu, Phys. Rev. D 82, 034023 (2010)

35. H.W. Ke, X.Q. Li, Y.L. Shi, Phys. Rev. D 87, 054022 (2013)

36. H.W. Ke, X. Q. Li, X. Liu, arXiv:1002.1187

37. J. Ferretti, E. Santopinto, Phys. Rev. D 90, 094022 (2014)

38. J. Ferretti, G. Galata, E. Santopinto, Phys. Rev. D 90, 054010 (2014)

39. Y. Lu, M.N. Anwar, B.S. Zou, Phys. Rev. D 94, 034021 (2016)

40. F. Fazio, Phys. Rev. D 79, 054015 (2009)

41. J. Segovia, P.G. Ortega, D.R. Entem, F. Fernàndez, Phys. Rev. D 93, 074027 (2016)

42. S. Godfrey, K. Moats, Phys. Rev. D 92, 054034 (2015)

43. P.A. Zyla et al., Prog. Theor. Exp. Phys. 2020, 083 C01 (2020)

44. W. Lucha, F. Schöberl, D. Gromes, Phys. Rep. (Rev. Sect. Phys. Lett.) 4, 200 (2018)

45. W. Kwong, J.L. Rosner, C. Quigg, Annu. Rev. Nucl. Part. Sci. 37, 325 (1987)

46. N. Brambilla et al., Eur. Phys. J. C 71, 1534 (2011)

47. J.J. Dudek, R.G. Edwards, N. Mathur, D.G. Richards, Phys. Rev. D 77, 034501 (2008)

48. S. Meinel, Phys. Rev. D 79, 094501 (2009)

49. A. Gray, I. Allison, C.T.H. Davies, E. Gulez, G.P. Lepage, J. Shigemitsu, M. Wingate, Phys. Rev. D 72, 094507 (2005)

50. T. Burch et al., Phys. Rev. D 81, 034508 (2010)

51. S. Meinel, Phys. Rev. D 82, 114502 (2010)

52. R. Lewis, R. Woloshyn, Phys. Rev. D 85, 114509 (2012)

53. M. Wurtz, R. Lewis, R.M. Woloshyn, Phys. Rev. D 92, 054504 (2015)

54. R. Dowdall et al., Phys. Rev. D 85, 054509 (2012)

55. E. Eitchten, K. Gottfried, T. Kinoshita, J.B. Kogut, K.D. Lane, T.M. Yan, Phys. Rev. Lett. 34, 369 (1975)

56. A.A. Belavin, A.M. Polyakov, A.S. Schwartz, Y.S. Tyupkin, Phys. Lett. B 59, 85 (1975)

57. U.T. Yakhshiev, H.C. Kim, B. Turimov, M.M. Musakhanov, Chin. Phys. C 41, 083102 (2017)

58. T. Schäfer, E.V. Shuryak, Instantons in QCD. Rev. Mod. Phys. 70(2) (1998)

59. F. Wilczek, A. Zee, Phys. Rev. Lett. 40, 83 (1978)

60. C.G. Callan, R.F. Dashen, D.J. Gross, F. Wilczek, A. Zee, Phys. Rev. D 18, 4684 (1978)

61. E. Eichten, F. Feinberg, Phys. Rev. D 23, 2724 (1981)

62. D. Diakonov, V.Y. Petrov, P.V. Pobylista, Phys. Lett. B 226, 372 (1989)

63. D. Diakonov, V.Y. Petrov, Nucl. Phys. B 245, 259 (1984)

64. D. Diakonov, Prog. Part. Nucl. Phys. 51, 173 (2003), and references therein

65. P. Pobylitsa, Phys. Lett. B 226, 387 (1989), and references therein

66. D. Diakonov, V.Y. Petrov, Nucl. Phys. B 272, 457 (1986)

67. D. Diakonov, Prog. Part. Phys. Lett. B 226 (1989)

68. E.V. Shuryak, Nucl. Phys. B 203, 93 (1982)

69. H.-Ch. Kim, M. Musakhanov, M. Siddikov, Phys. Lett. B 633 , 701 (2006) 
70. K. Goeke, H.-Ch. Kim, M. Musakhanov, M. Siddikov, Phys. Rev. D 76, 116007 (2007)

71. K. Goeke, M. Musakhanov, M. Siddikov, Phys. Rev. D 76, 076007 (2007)

72. T.A. DeGrand, Phys. Rev. D 64, 094508 (2001)

73. P. Faccioli, T.A. DeGrand, Phys. Rev. Lett. 91, 182001 (2003)

74. R. Van Royen, V.F. Weisskopf, Nuovo Cim A 50, 617 (1967)

75. E. Braaten, S. Fleming, Phys. Rev. D 52, 181 (1995)

76. A.V. Berezhnoy, V.V. Kiselev, A.K. Likhoded, Z. Phys. A 356, 89 (1996)

77. P.C. Vinodkumar, K.B. Vijayakumar, S.B. Khadkikar, Pramana J. Phys. 39, 47 (1992)

78. A.P. Monterio, K.B. Vijayakumar, Nat. Sci. 2, 1292 (2010)

79. J.L. Rosner et al. (CLEO Colloboration), Phys. Rev. Lett. 96, 092003 (2006)

80. S. Bhatnagar, S. Li, J. Phys. G Nucl. Part. Phys. 32, 949 (2006)

81. A. Badalyan, B. Ioffe, A.V. Smilga, Nucl. Phys. B 281, 85 (1987)

82. W. Kwong, P.B. Mackenzie, R. Rosenfeld, Phys. Rev. D 37, 3210 (1988)

83. R. Barbieri, R. Gatto, R. Kögerler, Z. Kunszt, Phys. Lett. B 57, 455 (1975)

84. V.A. Novikov, L.B. Okun, M.A. Shifman, A.I. Vainshtein, M.B. Voloshin, V.I. Zakharov, Phys. Rep. 41, 1 (1978)

85. J.P. Lansberg, T.N. Pham, Phys. Rev. D 79, 094016 (2009)

86. R. Barbieri, M. Caffo, R. Gatto, E. Remiddi, Nucl. Phys. B 192, $61(1981)$

87. G. Belanger, P. Moxhay, Phys. Lett. B 199, 575 (1987)

88. S.F. Radford, W.W. Repko, Phys. Rev. D 75, 074031 (2007)

89. T.A. Lahde, Nucl. Phys. A 714, 183 (2003)

90. B. Colquhoun, R.J. Dowdall, C. Davies, K. Hornbosteland, G.P. Lepage, Phys. Rev. D 91(7), 074514 (2015)

91. G.L. Wang, Phys. Lett. B 633, 492 (2006)

92. B. Patel, P.C. Vinodkumar, J. Phys. G Nucl. Part. 36, 035003 (2009)

93. T. Bhavsar, M.N. Shah, P.C. Vinodkumar, Eur. Phys. J. C 78, 227 (2018)

94. K. Bhaghyesh, B. Vijaya Kumar, A.P. Monterio, Phys. G. Nucl. Part. 38, 085001 (2011)

95. N.R. Soni, B.R. Joshi, R.P. Shah, H.R. Chauhan, J.N. Pandya, Eur. Phys. J. C 78, 592 (2018)
96. A. Krassnigg, M. Gomez-Rocha, T. Hilger, J. Phys. Conf. Ser. 742, 012032 (2016)

97. H. Negash, S. Bhatnagar, Int. J. Mod. Phys. E 25(8), 1650056 (2016)

98. C. McNielle, C. Davies E. Follana, K. Hornbostel, G.P. Lepage (HPQCD Collab.), Phys. Rev. D 86, 074503 (2012). arXiv:1207.0994 [he-lat]

99. E. Veli Veliev, K. Azizi, H. Sundu, N. Aksit, J. Phys. G Nucl. Part. 39, 015002 (2012)

100. S.F. Radford, W.W. Repko, Nucl. Phys. A 865, 69 (2011)

101. M. Shah, A. Parmar, P.C. Vinodkumar, Phys. Rev. D 86, 034015 (2012)

102. B.Q. Li, K.T. Chao, Commun. Theor. Phys. 52, 653 (2009)

103. D. Ebert, R.N. Faustov, V.O. Galkin, Mod. Phys. Lett. A 18, 601 (2003)

104. J.T. Laverty, S.F. Radford, W.W. Repko, arXiv:0901.3917 (2009)

105. A. Parmar, B. Patel, P.C. Vinodkumar, Nucl. Phys. A 848, 299 (2010)

106. S.N. Gupta, J.M. Johnson, W.W. Repko, Phys. Rev. D 54, 2075 (1996)

107. C.S. Fischer, S. Kubrak, R. Williams, Eur. Phys. J. A 51, 10 (2015)

108. D. Ebert, R.N. Faustov, V.O. Galkin, Eur. Phys. J. C 71, 1825 (2011)

109. W.J. Deng, H. Liu, L.C. Gui, X.H. Zhong, Phys. Rev. D 95, 074002 (2017)

110. M. Shah, B. Patel, P.C. Vinodkumar, Eur. Phys. J. C 76, 36 (2016)

111. M. Shah, B. Patel, P.C. Vinodkumar, Phys. Rev. D 93, 094028 (2016)

112. M. Shah, B. Patel, P.C. Vinodkumar, Phys. Rev. D 90, 014009 (2014)

113. W. Kwong, J.L. Rosner, Phys. Rev. D 38, 279 (1988)

114. A. Bradley, A. Khare, Z. Phys. C Part. Fields 8, 131 (1981)

115. F.E. Close, An Introduction to Quarks and Partons (Academic Press, London, 1979)

116. R. Bruschini, P. Gonzalez, Phys. Rev. D 100, 074001 (2019)

117. V.M. Abazov et al. (DØ), Phys. Rev. D. 86, 031103 (2012)

118. G. Aad et al. (ATLAS Collaboration), Phys. Rev. Lett. 108, 152001 (2012) 